\title{
A Subzone Reconstruction Algorithm for Efficient Staggered Compatible Remapping
}

\author{
D. P. Starinshak ${ }^{\mathrm{a}}$, J. M. Owen ${ }^{\mathrm{b}}$ \\ ${ }^{a}$ Lawrence Livermore National Laboratory, AX Division, M/S L-38, P.O. Box 808, \\ Livermore, CA 94550 \\ ${ }^{b}$ Lawrence Livermore National Laboratory, AX Division, M/S L-38, P.O. Box 808, \\ Livermore, CA 94550
}

\begin{abstract}
Staggered-grid Lagrangian hydrodynamics algorithms frequently make use of subzonal discretization of state variables for the purposes of improved numerical accuracy, generality to unstructured meshes, and exact conservation of mass, momentum, and energy. For Arbitrary Lagrangian-Eulerian (ALE) methods using a geometric overlay, it is difficult to remap subzonal variables in an accurate and efficient manner due to the number of subzone-subzone intersections that must be computed. This becomes prohibitive in the case of 3D, unstructured, polyhedral meshes. A new procedure is outlined in this paper to avoid direct subzonal remapping. The new algorithm reconstructs the spatial profile of a subzonal variable using remapped zonal and nodal representations of the data. The reconstruction procedure is cast as an underconstrained optimization problem. Enforcing conservation at each zone and node on the remapped mesh provides the set of equality constraints; the objective function corresponds to a quadratic variation per subzone between the values to be reconstructed and a set of target reference values. Numerical results for various pure-remapping and hydrodynamics tests are provided. Ideas for extending the algorithm to staggered-grid radiation-hydrodynamics are discussed as well as ideas for generalizing the algorithm to include inequality constraints.
\end{abstract}

Keywords: Remapping, ALE methods

Email addresses: starinshak1@llnl.gov (D. P. Starinshak), mikeowen@llnl.gov (J. M. Owen) 


\section{Background}

In the following sections, we introduce the problem of remapping subzonal quantities in the context of staggered-mesh discretizations. We define the subzonal spatial discretization of interest to our method, highlight specific algorithms which employ this discretization, and describe the constraints and accuracy requirements of these algorithms.

\subsection{Subzonal Discretization}

The principle application of this paper is the use of a geometric overlaybased remap with the spatially-staggered, Lagrangian hydrodynamics algorithm outlined in [2]. The hydrodynamic discretization of [2] derives a number of important properties - for instance, conservation, generality to unstructured grids, and improved accuracy - from the discretization of state variables on subzonal mesh elements.

The thrust of this paper is an efficient algorithm for reconstructing subzonal quantities (in particular, discrete masses) by remapping aggregate zonal and nodal versions of these quantities. The approach is applicable so long as the primary physics discretization is centered on zonal and/or nodal volumes, as is the case for the staggered Lagrangian hydrodynamic discretization we study here.

The ideas of this paper are expressed in terms of staggered-grid hydrodynamics, but the reconstruction methodology can be generalized to other types of discretization that employ subzonal quantities. One natural extension is the calculation of sub-grid temperatures following an overlay remap for the purposes of flux-limited diffusion (FLD) radiation-hydrodynamics on unstructured grids [27, 28, 29].

\subsection{Notation}

Each zone is subdivided into subzonal elements, which we occasionally refer to as corners and index by $c$. Each corner is a polyhedron in 3D or a quadrilateral in $2 \mathrm{D}$ and is uniquely identified with a single zone $z_{c}$ and node $n_{c}$ on the Lagrangian mesh. The vertices of corner $c$ consist of the centroid $\mathbf{x}_{z_{c}}$ of its zone, the position $\mathbf{x}_{n_{c}}$ of its node, and the centers of each of the faces of zone $z_{c}$ that touch $n_{c}$. Figure 1 illustrates the corner discretization in $2 \mathrm{D}$ and $3 \mathrm{D}$. 
In terms of finite volumes, the corner $c$ occupies the intersection of zone element $z_{c}$ on the primary mesh and node elements $n_{c}$ on the dual mesh. Element volumes for zones $V_{z}$ and nodes $V_{n}$ are defined by summing neighboring corner volumes $V_{c}$ :

$$
V_{z}=\sum_{c(z)} V_{c} \quad \text { and } \quad V_{n}=\sum_{c(n)} V_{c} .
$$

Here $c(z)$ denotes the set of corners inside zone $z$, and $c(n)$ denotes the set of corners touching node $n$.
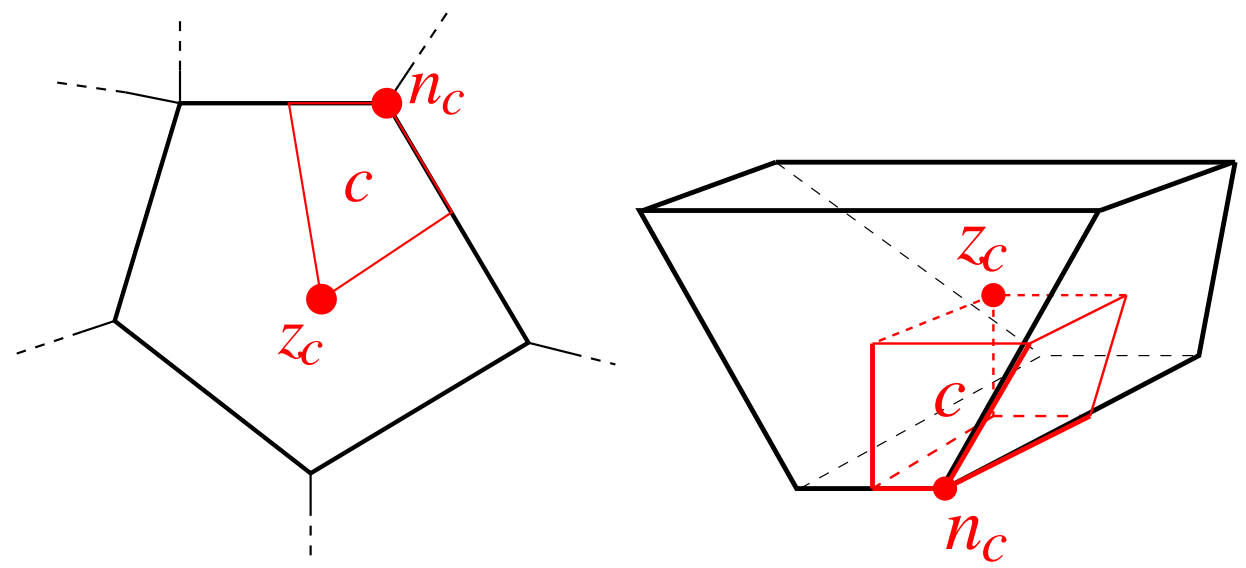

Figure 1: Corner subvolumes ( $c$, in red) of a 2D (left) and 3D (right) zone.

\subsection{Subzonal Densities and Compatible Hydrodynamics}

We consider subzonal discretizations in the context of conservative hydrodynamics. We assume spatially-staggered variables: kinematic variables of velocity and kinetic energy at nodes; thermodynamic variables of internal energy at zone centers.

The compatible formalism in [2] derives a consistent discretization of the evolution equations for fluid momentum (on nodes) and energy (on zones) using a subzonal discretization of the underlying mass density. The zonal and nodal representations of mass that define discrete energy and momenta respectively are coarsened versions of the sub-grid mass distribution on corners:

$$
m_{z}=\sum_{c(z)} m_{c} \quad \text { and } \quad m_{n}=\sum_{c(n)} m_{c} .
$$


Here $m_{z}, m_{n}$, and $m_{c}$ denote discrete masses centered at zone $z$, node $n$, and corner $c$ respectively. The corresponding densities satisfy

$$
\rho_{\alpha}=m_{\alpha} / V_{\alpha}, \quad \alpha=z, n, c .
$$

\subsection{Problem Statement}

The existence of discrete state variables at zones, nodes, and subzones presents us with a considerable challenge in the context of Arbitrary LagrangianEulerian (ALE) methods: in order to remap state variables from one mesh to another in a conservative fashion, accurate subzonal information must be maintained. The problem has been interpreted in terms of both fluxbased and overlay-based remaps. Owen and Shashkov describe a flux-based strategy in [21] that computes consistent subzonal fluxes around nodes to preserve mass and momentum conservation. Subzonal fluxes are computed in a manner that reduces the local variation with respect to an optimal reference flux. Unfortunately, the flux based algorithm of [21] implicitly assumes the topology of the mesh is not changing, only the geometry. Extensions to the flux-based remap have been presented that do not assumed a fixed topology, for instance $[7,10]$, but the problem of remapping staggered or subzonal data has not yet been addressed. For this paper, we focus on geometric overlay-based remapping. The ideas presented build on those in [21].

In an overlay remap, state variables are mapped from one spatial partition (termed the donor mesh) to a second partition (target mesh) based on geometrical intersections between donor and target elements. The remap is generic in the sense that the topologies of the donor and target may be entirely unrelated.

For cell-centered discretizations, all state variables are collocated to zone centers; only zone-zone intersections need to be computed in the overlay. The computational complexity increases for staggered discretizations due to the fact that variables are centered at both zones and nodes. Zone-zone intersections are computed to remap quantities such as internal energy; nodenode (i.e. dual mesh) intersections are computed to remap quantities such as momentum and kinetic energy.

Intersection operations can impose steep memory requirements and become computationally expensive, particularly if (i) mesh elements increase in complexity, as with unstructured, polygonal meshes (for instance, ReALE [13]); (ii) additional physics is coupled into the problem, thus increasing the number remapped variables; or (iii) the problem increases in dimensionality. 
When state variables are discretized at mesh subzones, their information is lost during a geometric overlay if mesh intersections are limited to zones and nodes only. For compatible hydrodynamics, this means losing the very conservation property provided by the discretization in the first place. For staggered radiation-hydrodynamics, this means losing information on intrazonal variation and reducing the accuracy of fluid-radiation coupling terms.

We recognize two general strategies for maintaining subzonal state information: (i) remap subzonal information directly from donor mesh to target mesh or (ii) reconstruct subzonal data using information at neighboring zones and nodes. This paper describes a new algorithm for strategy (ii). We first summarize the state-of-the-art for strategy (i).

An accurate and efficient implementation of strategy (i) is described in [14] and successfully applied to staggered ReALE hydrodynamics calculations in [13]. The algorithm is efficient in the sense that geometric intersections are only computed between subzones. All variables are collocated to neighboring subzones to accomplish this. The collocation process, referred to as "gathering," computes subzonal values for all zonal and nodal quantities. After the remap, the gather stage is reversed in a process referred to as "scattering," and consistent zonal and nodal discretizations are recovered from their remapped state on subzones. The gather-remap-scatter process is designed to be conservative, reversible, and second-order accurate. The authors employ a local repair procedure based on $[3,16,18]$ to ensure the remap is also monotonic.

Remapping subzones to subzones is an intuitive strategy, but it unfortunately does not solve the problem of computational complexity. The number of element intersections scales with the total number of elements in both donor and target meshes. For the simple case of quad zones in 2D, the number of subzone-subzone intersections is still twice the sum of both zone-zone and node-node intersections in pure element count. For arbitrary polyhedral zones in 3D, the number of subzones becomes much greater than the sum of zones and nodes together. In addition to cost, the direct subzonal remap also lacks a straightforward extension to multi-material remapping. The ultimate goal of this work is to perform conservative overlay remapping with multiple materials in both $2 \mathrm{D}$ and 3D. To that end, direct subzonal remapping is not an ideal solution.

We are fortunate in that the hydrodynamic equations are differenced on the nodal and zonal volumes. This suggests that carefully remapping the nodal and zonal quantities and conservatively reconstructing subzonal values 
from them should be adequate to maintain the overall accuracy of the hydrodynamic method. The reconstruction algorithm we propose is described in detail in the following sections. Our approach corresponds to solving a constrained optimization problem in which discrete conservation conditions serve as linear constraints on the subzonal field of interest.

\section{Reconstruction Algorithm}

This section describes the algorithm for reconstructing subzonal information from discrete data at zones and nodes. We focus on reconstructing subzonal mass density for the purpose of remapping staggered-grid hydrodynamics variables. In general, the algorithm applies to any subzonal quantity that is conserved by volume.

The initial conditions of the algorithm consist of post-remap mass and volume data obtained from an overlay remap. Using (2), discrete zone and node masses $\widetilde{m}_{z}$ and $\widetilde{m}_{n}$ respectively are formed on the pre-remap mesh. The overlay returns remapped zone masses $m_{z}$ and node masses $m_{n}$. (Here we denote pre-remap values with $\widetilde{\cdot}$ and post-remap values without.) The number of zones, nodes, and subzones on the post-remap mesh are $N_{z}, N_{n}$, and $N_{c}$ respectively, with subzonal volumes denoted $V_{c}$. The unknowns are the $N_{c}$ discrete subzonal mass densities, denoted $\rho_{c}$.

We solve for subzonal densities satisfying the following conditions:

(i) The sum of subzonal masses in a zone recovers the zonal mass.

(ii) The sum of subzonal masses around a node recovers the nodal mass.

(iii) Each subzonal density is as close as possible to a reference state.

We interpret the statements above as a constrained optimization problem. Statements $(i)$ and $(i i)$ are linear constraints on the set of possible corner density values. Statement (iii) defines the variation with respect to a desired density field.

Using (1), (2), and (3), constraints $(i)$ and (ii) are written in terms of local corner information:

$$
(i): \quad m_{z}=\sum_{c(z)} m_{c}=\sum_{c(z)} V_{c} \rho_{c}=\rho_{z} \sum_{c(z)} V_{c} \Longrightarrow \sum_{c(z)} V_{c}\left(\rho_{c}-\rho_{z}\right)=0 .
$$


and

$$
\text { (ii) : } m_{n}=\sum_{c(n)} m_{c}=\sum_{c(n)} V_{c} \rho_{c}=\rho_{n} \sum_{c(n)} V_{c} \Longrightarrow \sum_{c(n)} V_{c}\left(\rho_{c}-\rho_{n}\right)=0 .
$$

For statement (iii), we define a positive relative variation per subzone

$$
\left(\rho_{c} / \mu_{c}-1\right)^{2}
$$

where $\mu_{c}$ denotes some optimal reference state. Summing over all subzones defines a global functional

$$
\text { (iii) : } \quad \frac{1}{2} \sum_{c}\left(\frac{\rho_{c}}{\mu_{c}}-1\right)^{2}
$$

To summarize, we cast $(i)-(i i i)$ as a constrained optimization problem:

$$
\begin{array}{ll}
\text { Given: } & \rho_{z}, \rho_{n}, V_{c} \\
\text { Minimize: } & \frac{1}{2} \sum_{c}\left(\frac{\rho_{c}-\mu_{c}}{\mu_{c}}\right)^{2} \\
\text { Subject to: } & \begin{cases}\sum_{c(z)} V_{c}\left(\rho_{c}-\rho_{z}\right)=0, & \forall z \\
\sum_{c(n)} V_{c}\left(\rho_{c}-\rho_{n}\right)=0, & \forall n\end{cases}
\end{array}
$$

We describe possible forms of $\mu_{c}$ in section 3 .

We solve (7) by the technique of Lagrange multipliers. Define multipliers $\lambda_{z}$ and $\lambda_{n}$ corresponding to each zone constraint (4) and node constraint (5) respectively and define the global Lagrangian functional

$L\left(\rho_{c}, \lambda_{z}, \lambda_{n}\right)=\frac{1}{2} \sum_{c}\left(\frac{\rho_{c}-\mu_{c}}{\mu_{c}}\right)^{2}+\sum_{z} \lambda_{z} \sum_{c(z)} V_{c}\left(\rho_{c}-\rho_{z}\right)+\sum_{n} \lambda_{n} \sum_{c(n)} V_{c}\left(\rho_{c}-\rho_{n}\right)$.

The gradient of $L$ is

$$
\begin{aligned}
\frac{\partial L}{\partial \rho_{c}} & =\frac{\rho_{c}-\mu_{c}}{\mu_{c}^{2}}+V_{c} \lambda_{z_{c}}+V_{c} \lambda_{n_{c}} \\
\frac{\partial L}{\partial \lambda_{z}} & =\sum_{c(z)} V_{c}\left(\rho_{c}-\rho_{z}\right) \\
\frac{\partial L}{\partial \lambda_{n}} & =\sum_{c(n)} V_{c}\left(\rho_{c}-\rho_{n}\right)
\end{aligned}
$$


where, again, $z_{c}$ is the unique zone containing corner $c$, and $n_{c}$ is the unique node touching corner $c$. Setting $\partial L / \partial \rho_{c}=0$ gives an expression for $\rho_{c}$ in terms of a perturbation on its reference value:

$$
\rho_{c}=\mu_{c}\left(1-\mu_{c} V_{c}\left(\lambda_{z_{c}}+\lambda_{n_{c}}\right)\right) .
$$

Solving for the fixed points $\partial L / \partial \lambda_{n}=0$ and $\partial L / \partial \lambda_{z}=0$ corresponds to a linear system with $N_{z}+N_{n}$ unknowns. Thankfully, we can factor this system and reduce the problem to solving for only $N_{z}$ unknowns. Substituting (10) into $\partial L / \partial \lambda_{n}=0$ gives an expression for $\lambda_{n}$ in terms of values of $\lambda_{z}$ at zones neighboring node $n$ :

$$
\lambda_{n}=-\frac{\sum_{c(n)}\left(\mu_{c} V_{c}\right)^{2} \lambda_{z_{c}}+\sum_{c(n)}\left(\rho_{n}-\mu_{c}\right) V_{c}}{\sum_{c(n)}\left(\mu_{c} V_{c}\right)^{2}}=-\frac{\sum_{c(n)} w_{c} \lambda_{z_{c}}+\Delta m_{n}}{W_{n}} .
$$

For convenience, we define corner weights based on the square of the reference mass

$$
w_{c}=\left(\mu_{c} V_{c}\right)^{2}
$$

as well as zone and node weights

$$
W_{z}=\sum_{c(z)} w_{c} \quad \text { and } \quad W_{n}=\sum_{c(n)} w_{c} .
$$

We also define zonal and nodal mass differences relative to the reference state:

$$
\begin{aligned}
& \Delta m_{z}=m_{z}-\sum_{c(z)} \mu_{c} V_{c}=\sum_{c(z)}\left(\rho_{z}-\mu_{c}\right) V_{c} \\
& \Delta m_{n}=m_{n}-\sum_{c(n)} \mu_{c} V_{c}=\sum_{c(n)}\left(\rho_{n}-\mu_{c}\right) V_{c}
\end{aligned}
$$

Finally, setting $\partial L / \partial \lambda_{z}=0$ and substituting (10) and (11), gives a linear expression for each zonal Lagrange multiplier $\lambda_{z}$ :

$$
W_{z} \lambda_{z}-\sum_{n(z)} \frac{w_{c(z, n)}}{W_{n}} \sum_{c(n)} w_{c} \lambda_{z_{c}}=\sum_{n(z)} \frac{w_{c(z, n)}}{W_{n}} \Delta m_{n}-\Delta m_{z},
$$


where $n(z)$ denotes the set of nodes around zone $z$, and $c(z, n)$ denotes the unique subzone inside zone $z$ and touching node $n$.

The unknowns in (15) are the $N_{z}$ zonal Lagrange multipliers $\lambda_{z}$. We write (15) as a linear system of the form

$$
\left(\mathbb{W}_{z}+\mathbb{Y}\right) \lambda=\mathbf{b}
$$

Here $\lambda$ is the vector of Lagrange multipliers $\left\{\lambda_{z}\right\}_{n=1}^{N_{z}}$, b is a length $N_{z}$ vector of known quantities, $\mathbb{W}_{z}$ is an $N_{z} \times N_{z}$ diagonal matrix consisting of zone weights (13), and $\mathbb{Y}$ is a sparse $N_{z} \times N_{z}$ matrix. The $i$-th entry of $\mathbf{b}$ corresponds to zone $z_{i}$ :

$$
b_{i}=\sum_{n\left(z_{i}\right)} \frac{w_{c\left(z_{i}, n\right)}}{W_{n}} \Delta m_{n}-\Delta m_{z_{i}} .
$$

The $(i, j)$-th entry of $\mathbb{Y}$ corresponds to zone pair $\left(z_{i}, z_{j}\right)$ :

$$
Y_{i, j}=-\sum_{n\left(z_{i}, z_{j}\right)} \frac{w_{c\left(z_{i}, n\right)} w_{c\left(z_{j}, n\right)}}{W_{n}},
$$

where $n\left(z_{i}, z_{j}\right)$ is the set of nodes shared by both zones $z_{i}$ and $z_{j}$. Matrix $\mathbb{Y}$ is sparse in the sense that, if $z_{i}$ and $z_{j}$ do not share any nodes, $Y_{i, j}=0$. Alternatively, one can interpret the individual rows of matrix $\mathbb{Y}$ using the topology of the mesh: the nonzero entries for row $i$ correspond to the mesh stencil around $z_{i}$ of all zones which share at least one node with $z_{i}$ (see fig. 2).

It is instructive to write matrices $\mathbb{W}_{z}$ and $\mathbb{Y}$ as products of geometrical sub-matrices based on the mesh connectivity. The sub-matrices are weighted by subzone volume for notational convenience. Define

$$
\begin{aligned}
& \mathbb{V}_{c z}= \begin{cases}V_{c} & , \text { subzone } c \text { is contained in zone } z \\
0 & , \text { o.w. }\end{cases} \\
& \mathbb{V}_{c n}= \begin{cases}V_{c} & , \text { subzone } c \text { touches node } n \\
0 & , \text { o.w. }\end{cases}
\end{aligned}
$$

where $\mathbb{V}_{c z}$ is size $N_{c} \times N_{z}$, and $\mathbb{V}_{c n}$ is size $N_{c} \times N_{n}$. Similarly, write diagonal matrices $\mathbb{W}_{z}=\operatorname{diag}\left(\left\{W_{z}\right\}_{z}\right), \mathbb{W}_{n}=\operatorname{diag}\left(\left\{W_{n}\right\}_{n}\right)$, and $\mathbb{M}_{c}=\operatorname{diag}\left(\left\{\mu_{c}\right\}_{c}\right)$ for zone weights, node weights, and reference values respectively. Then,

$$
\mathbb{W}_{z}=\mathbb{V}_{c z}^{T} \mathbb{M}_{c}^{2} \mathbb{V}_{c z} \quad \text { and } \quad \mathbb{W}_{n}=\mathbb{V}_{c n}^{T} \mathbb{M}_{c}^{2} \mathbf{V}_{c n}
$$




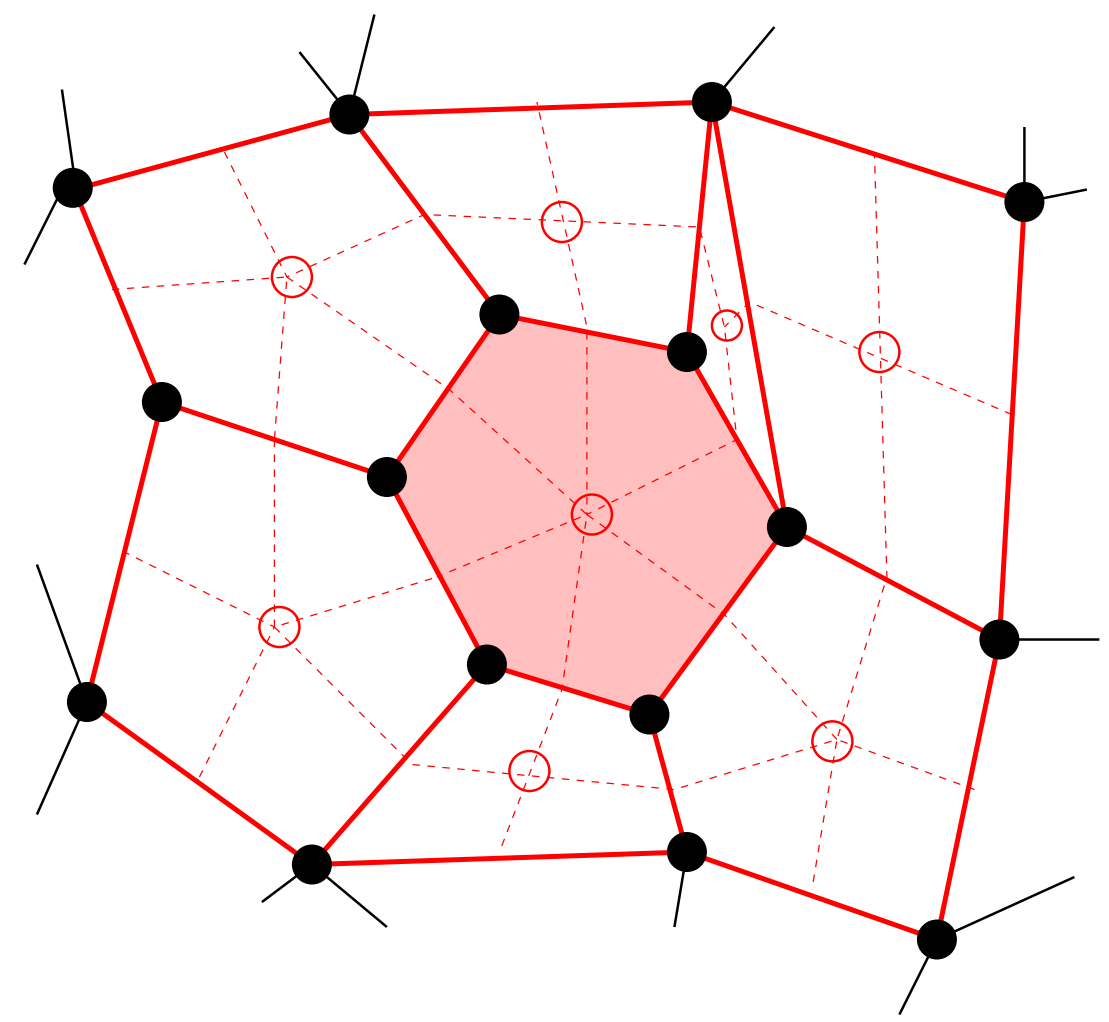

Figure 2: An example of the stencil of zones that share at least one node with with the shaded $2 \mathrm{D}$ polygonal zone.

by construction and similarly

$$
\mathbb{Y}=-\mathbb{V}_{c z}^{T} \mathbb{M}_{c}^{2} \mathbb{V}_{c n} \mathbb{W}_{n}^{-1} \mathbb{V}_{c n}^{T} \mathbb{M}_{c}^{2} \mathbb{V}_{c z}
$$

Finally, define

$$
\mathbb{A}=\mathbb{W}_{z}+\mathbb{Y}=\mathbb{V}_{c n}^{T} \mathbb{M}_{c}\left(\mathbb{I}_{c}-\mathbb{M}_{c} \mathbb{V}_{c z} \mathbb{W}_{z}^{-1} \mathbb{V}_{c z}^{T} \mathbb{M}_{c}\right) \mathbb{M}_{c} \mathbb{V}_{c n}
$$

The matrix form of (15) is given by the system

$$
\mathbb{A} \lambda=\mathbf{b} .
$$

A potential wrinkle involved with solving (23) is that $\mathbb{A}$ is singular. In general, the constrained optimization problem in (7) is underdetermined: the 
combined number of constraints (4) and (5) are fewer than the total number of unknowns (i.e. $N_{c} \geq N_{z}+N_{n}$ ). A unique set of constrained subzonal densities which minimize (6) does not exist.

One option for solving (23) is singular value decomposition (SVD) of $\mathbb{A}$. While this method does arrive at the best-fit solution to (23), it is not ideal for very large problems. An alternate method that is faster and equally stable is a preconditioned linear solve. For this paper, we use the algebraic multi-grid (AMG) preconditioner and GMRES solver in the parallel linear algebra package Hypre [8]. The fact that $\mathbb{A}$ is singular simply means Hypre cannot do a direct matrix inversion for the coarsest AMG level.

The reason we do not have to resort to a full SVD solve is because the null space of $\mathbb{A}$ is of dimension 1 . A preconditioned iterative solver will converge to the minimizing solution provided the initial guess is outside the null space of $\mathbb{A}$. This is easily accomplished by setting the initial guess to be non-constant. Justification for these points is provided in appendix A.

Remark: Penalty methods provide a simple alternative to using Lagrange multipliers for constrained optimization. Instead of enforcing linear constraints, additional penalty terms can be added to (6) to discourage unsatisfactory states, such as negative or non-monotonic values, from appearing in the subzonal density field. Unfortunately, penalty methods do not satisfy equality constraints exactly - only in an iterative limit. This is undesirable for our purposes given our strict consistency conditions between zones and nodes to satisfy mass conservation.

Remark: Incorporating inequality constraints into (7) is possible through the formalism of Karush-Kuhn-Tucker and complementary slackness [9, 11]. Inequality constraints may be used to enforce positivity in the resulting subzonal density solution or, stronger still, monotonicity with respect to neighboring zonal and nodal density values. See appendix $\mathrm{C}$ for a thorough discus-

sion of these ideas as well as generalization of the reconstruction algorithm to incorporate inequality constraints.

\section{Reference State}

The reference state in (6) defines a locally optimal value for a given subzone. Intuitively, the reference state should depend on the post-remap density field local to each subzone. We assume: 
- $\mu_{c}$ is a function of local zone density $\rho_{z_{c}}$ and local node density $\rho_{n_{c}}$.

- $\mu_{c}$ is bounded by the two local densities.

We consider six choices for $\mu_{c}$ in this article:

(i) Zone Density:

$\rho_{z_{c}}$

(ii) Node Density:

$\rho_{n_{c}}$

(iii) Arithmetic Average:

$\frac{\rho_{z_{c}}+\rho_{n_{c}}}{2}$

(iv) Harmonic Average:

$$
\frac{2 \rho_{z_{c}} \rho_{n_{c}}}{\rho_{z_{c}}+\rho_{n_{c}}}
$$

(v) Local Maximum:

$\max \left(\rho_{z_{c}}, \rho_{n_{c}}\right)$

(vi) Local Minimum:

$\min \left(\rho_{z_{c}}, \rho_{n_{c}}\right)$

Numerical tests comparing reference states for a variety of pure remapping problems are included in section 7 .

Remark: The choice of reference state may be generalized beyond what is considered in this article. For instance, a different reference state may be chosen per-subzone based on local smoothness of the zonal and nodal density fields. In regions near large discontinuities, reference values that compute strictly positive densities are desirable; in smooth regions, the local zone value may be desirable. Note also that (i)-(iv) may be combined into two general forms of the arithmetic and harmonic average,

$$
\mu_{c}=\alpha_{c} \rho_{z_{c}}+\left(1-\alpha_{c}\right) \rho_{n_{c}} \quad \text { and } \quad \mu_{c}=\frac{\rho_{z_{c}} \rho_{n_{c}}}{\alpha_{c} \rho_{n_{c}}+\left(1-\alpha_{c}\right) \rho_{z_{c}}},
$$

respectively, where $\alpha_{c} \in[0,1]$ smoothly tracks the contributions of zone and node densities in the reference state per-subzone. Analysis of (25) is a topic of future work.

\section{Repairing Subzonal Values}

For certain choices of reference state, the algorithm may minimize the objective function (6) but compute subzonal values that are negative. Reconstructed values may also be non-monotonic, i.e. straying outside the bounds 
set by their local zonal and nodal values. This is most likely to occur in regions with large discrepancies between zonal and nodal values, for instance when remapping a density field having steep discontinuities.

The reason for this is the extra degrees of freedom in the problem. The number of zonal and nodal constraints is less than the total number of subzones. Only the value of the reference state in each subzone may be used to drive the reconstructed values to ones that preserve positivity, monotonicity, etc.

In the spirit of the monotonicity-repairing methods of $[3,16,17,18]$, we consider a simple corrective procedure for repairing negative reconstructed values. Subzonal mass is redistributed within each zone in a manner that preserves zone mass exactly (4). The compromise involved in forcing positivity is a small error in node mass conservation (5).

The local repair process acts on zones containing at least one negative subzonal density. For zone $z$ having remapped mass $m_{z}$, let $\mathcal{C}^{-}$denote the set of subzones having negative reconstructed densities. Here $\mathcal{C}^{-}$is a strict subset of $c(z)$ since the remap itself ensures $m_{z}>0$.

In zone $z$ :

1. Sum the total negative subzonal mass within the zone:

$$
M^{-}=\sum_{c \in \mathcal{C}^{-}}\left|\rho_{c} V_{c}\right| .
$$

2. Replace all negative subzonal densities with a small positive constant value $\epsilon$ chosen to be smaller than the minimum density globally:

$$
\rho_{c} \longleftarrow \epsilon \forall c \in \mathcal{C}^{-}
$$

The amount of mass added to the zone by this replacement is

$$
M_{\epsilon}=\sum_{c \in \mathcal{C}^{-}} \epsilon V_{c}
$$

For all numerical tests, we use $\epsilon=10^{-10}$.

3. Scale the remaining positive subzonal densities by the ratio

$$
\rho_{c} \longleftarrow\left(\frac{m_{z}-M_{\epsilon}}{m_{z}+M^{-}}\right) \rho_{c}, \quad \forall c \in c(z), c \notin \mathcal{C}^{-} .
$$


This is equivalent to subtracting a fraction of the total mass change $M^{-}+M_{\epsilon}$ in the zone from each positive subzone mass. The fraction per subzone is the ratio of its initial mass to total positive zone mass $m_{c} /\left(m_{z}+M_{-}\right)$. This ensures all subzone masses are positive and (4) is respected.

Unlike the repair methods of $[3,16,17,18]$, the correction outlined above is nonconservative. In the context of hydrodynamics, the ultimate consequence of redistributing subzonal mass within a zone is an error in momentum conservation. The size of this error scales with the total negative mass $M^{-}$and affects the discrete momentum values at each node around a corrected zone. Since some subzones gain mass and others lose it during the correction, the net effect on global momentum conservation has no definite sign. Furthermore, if velocity is constant, the correction will produce zero net momentum error.

It's important to point out that negative reconstructed values typically occur in the presence of steep discontinuities. They are rarely encountered in the context of hydrodynamic calculations because discontinuities - shocks, materials fronts, etc. - tend to spread across more than a single zone due to numerical diffusion. In our experience, local repair of negative values is seldom performed.

Remark: The small conservation error introduced by local repair is not significant for the types of problems we have considered. For the sake of completeness, we include two additional procedures for enforcing positivity that are fully conservative:

(i) Appendix B describes a global extension of the above local procedure. The global linear system in section 2 is modified by additional equality constraints imposed on subzones and solved a second time to remove negative values.

Although the resulting reconstruction is conservative, positivity is not guaranteed. Because (7) is globally coupled, new negative values may pop up elsewhere in the mesh to satisfy constraints (4) and (5) as well as the new set of imposed constraints. Experiments involving this procedure produced over-constrained problems, ultimately requiring the local, nonconservative repair procedure. 
(ii) Appendix $\mathrm{C}$ generalizes the constrained optimization problem to include inequality constraints. The method is fully conservative. Inequality constraints may also be chosen to enforce not only positivity but also monotonicity in the reconstructed subzonal field.

Future work on subzonal reconstruction will focus on imposing inequality constraints. Our implementation does not currently support them.

These algorithms are understandably more expensive than performing a local repair.

\section{Subzonal Pressures}

The subzonal reconstruction algorithm is well-optimized for use with the corner-based staggered compatible hydrodynamics algorithm of [2]. The discretization is sensitive to the total zonal and nodal masses but not to the individual subzonal values. As a result, the hydro algorithm performs well even if non-monotonicities creep into the reconstructed subzonal density field. The most important property to meet is that the subzonal masses sum to the correct remapped zonal and nodal values, which our algorithm ensures by construction.

The same cannot be said about subzonal densities that are used to compute subzonal pressures to serve as hourglass controls, as in [2]. Subzonal pressures computed from oscillatory subzonal mass densities can introduce numerical instabilities in the Lagrangian mesh. To avoid this complication, we use the anti-hourglassing filter of [4] instead of subzonal pressures to remove spurious grid motion in our hydro tests.

Remark: Reconstruction does not forbid us from taking advantage of subzonal pressures to improve mesh motion. Similar to [21], we could use a separate and distinctly simpler definition of corner mass density solely for computing subzonal pressures. This separate subzonal density would not contribute to the momentum equation and therefore would not have to meet the same stringent conservation properties as the reconstructed values. The idea in [21] may be extended to an overlay remap by considering the change in mass inside a zone before and after an overlay:

$$
m_{c}^{S P}= \begin{cases}\frac{V_{c}}{V_{z}} \Delta m_{z}, & \text { if zone mass increases } \\ \frac{m_{z}}{\widetilde{m}_{z}} m_{c}^{S P}, & \text { if zone mass decreases }\end{cases}
$$


where $m_{c}^{S P}$ indicates the subzonal pressure corner mass, $\Delta m_{z}=m_{z}-\widetilde{m}_{z}$ is the change in mass in zone $z$ during the remap. Again, $\sim$ denotes a pre-remap value, while post-remap values are bare. This definition will reproduce the remapped zonal mass from summing $m_{c}^{S P}$ in the zone, but not the remapped nodal masses. Such nodal reproduction is not necessary in the hourglass control and is therefore a perfectly acceptable compromise. The ability to use the subzonal corner pressure hourglass control will become important when we consider hydrodynamic examples on arbitrary polygonal grids. The kinematic filters of [4], which are specialized for quadrilaterals, are not appropriate for such calculations.

\section{Timing Comparison}

A major selling point of the reconstruction algorithm is that it avoids the expense of a direct subzone-to-subzone overlay. To justify this point, we have timed a series of pure remapping tests. We present serial and strong parallel scaling results for a mesh composed of quadrilaterals in table 1 and a mesh composed of packed hexagons in table 2. The quantity of interest is the percentage of time taken up by the reconstruction algorithm relative to the time to perform the geometric overlay. Although we do not have a direct subzone-to-subzone overlay to compare timing results, we can nevertheless compare how each method scales relative to the number of mesh elements taking part in the overlay.

For a mesh of $N$ quad elements, there are, on the order, $N$ nodes and $4 N$ subzones. Our timing results suggest that reconstruction accounts for approximately $15 \%$ of the total overlay cost. Therefore, a staggered-grid overlay with reconstruction scales with $1.15 \times 2 N=2.3 N$. By comparison, the subzone-to-subzone overlay scales with $4 N$ - the total number of subzones in the problem.

Similarly, for a mesh of $N$ packed hexagons, there are, on the order, $2 N$ nodes and $6 N$ subzones. Timing results indicate reconstruction adds an additional $20 \%$ to the total cost of the overlay. Therefore, a staggeredgird overlay with reconstruction scales with $3.6 \mathrm{~N}$, compared with $6 \mathrm{~N}$ for the direct subzone-to-subzone overlay.

\section{Numerical Results}

The numerical results in this section result from applying the corner reconstruction algorithms described in this paper to data remapped using an 


\begin{tabular}{ccc|cc|cc}
\hline$(N)$ & $(N)$ & $(4 N)$ & \multicolumn{2}{|c|}{ Serial Scaling } & \multicolumn{2}{c}{ Strong Parallel Scaling } \\
Zones & Nodes & Subzones & Procs & \% of Overlay & Procs & \% of Overlay \\
\hline 4096 & 4225 & 16384 & 1 & 12.3 & 1 & 12.3 \\
16384 & 16641 & 65536 & 1 & 12.7 & 4 & 15.1 \\
65536 & 66049 & 262144 & 1 & 13.7 & 16 & 17.3 \\
\hline
\end{tabular}

Table 1: Serial and strong parallel scaling results for a mesh of quadrilaterals. Timing data for the reconstruction algorithm is given relative to the time required to perform a staggered-grid geometric overlay.

\begin{tabular}{ccc|cc|cc}
\hline$(N)$ & $(2 N)$ & $(6 N)$ & \multicolumn{2}{c|}{ Serial Scaling } & \multicolumn{2}{c}{ Strong Parallel Scaling } \\
Zones & Nodes & Subzones & Procs & \% of Overlay & Procs & \% of Overlay \\
\hline 4257 & 8516 & 25284 & 1 & 18.8 & 1 & 18.8 \\
16705 & 33412 & 99716 & 1 & 19.6 & 4 & 20.2 \\
66177 & 132356 & 396036 & 1 & 22.5 & 16 & 24.8 \\
\hline
\end{tabular}

Table 2: Serial and strong parallel scaling results for a mesh of packed hexagons. Timing data for the reconstruction algorithm is given relative to the time required to perform a staggered-grid geometric overlay.

existing geometrical overlay package named Overlink [6]. Overlink uses linear reconstruction with gradient limiting to achieve second-order accuracy and monotonicity in the overlay. Arbitrary polyhedral intersections are computed using fast decomposition into tetrahedra. The full details of the algorithm are presented in [6]. For our purpose Overlink is a nice example of an existing geometric overlay remapping package intended for use with staggered data that we wish to generalize for use with subzonal corner data.

\subsection{Pure Remapping Tests}

In each test, we compute convergence of the reconstructed subzonal density values to their initial discrete values under $L_{1}$ norm. We compute the $L_{1}$ norm using the formula

$$
L_{1}=\frac{1}{M} \sum_{i=1}^{M}\left|a_{i}-b_{i}\right|,
$$


where $\left\{a_{i}\right\}_{i}$ and $\left\{b_{i}\right\}_{i}$ are arbitrary sequences of length $M$.

\subsubsection{Null Problem}

We begin by considering the null problem of overlaying a specified density field onto the same mesh. This is an exact operation using Overlink, thereby allowing us to test the accuracy of different choices of reference density in reconstructing an underlying subzonal density field. We consider two analytic density distributions - linear and discontinuous - and test the reconstruction algorithm on various discretizations of these distributions. Reconstruction is computed on $N \times N$ square meshes for $N \in\{8,16,32,64,128\}$.

\section{Test 1: Linear Density Field}

First is a linear density distribution. The continuous field is given by

$$
\rho(\mathbf{x})=\rho_{0}+\nabla \rho \cdot \mathbf{x}
$$

The field is discretized on each subzone by evaluating (27) at subzone center $\mathbf{x}_{c}$. Discrete zonal and nodal values are initialized by summing over subzones. In all subsequent tests, we use $\rho_{0}=10$ and $\nabla \rho=(1,3)$.

Figure 3 contains convergence curves for each of the six reference states. Dashed lines of slope 1, 2, and 3 illustrate the convergence rates of the various references. The results match expectations: the local minimum and maximum references are discontinuous functions of the local zonal and nodal values for each subzone; these references may only converge at first order. The zone density, node density, and harmonic average references are smooth functions and converge at second order. The harmonic average reference has smaller absolute error because the reference densities in each subzone are closer to the analytic distribution than the discrete zonal and nodal densities. The arithmetic average reference converges at third order. This is because the reconstruction only commits an error inside subzones on the boundary of the problem - a set having codimension 1 . The reference density at all interior subzones is equal to the analytic distribution.

\section{Test 2: Linear Density Field, Anti-Correlated Subzonal Field}

We use the same continuous field (27). Discrete zone densities $\rho_{z}$ are initialized by evaluating (27) at zone centers $\mathbf{x}_{z}$. A subzonal density distribution is chosen to have the opposite variation of the analytic field:

$$
\rho_{\text {sub }}(\mathbf{x})=\rho_{z}-f \nabla \rho \cdot\left(\mathbf{x}-\mathbf{x}_{z}\right), \quad \mathbf{x} \in\{\text { zone } z\}
$$




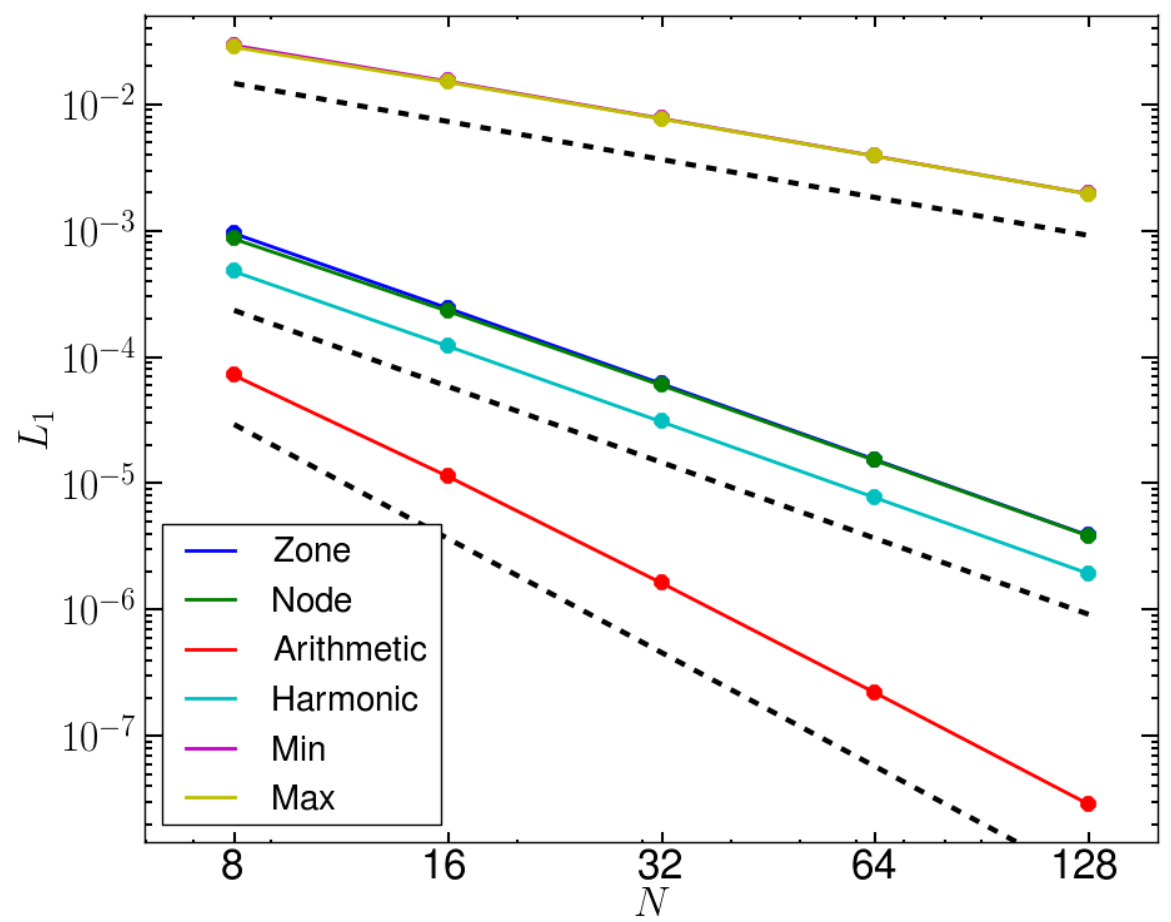

Figure 3: Null problem, linear field: $L_{1}$ convergence in subzonal density. Reference slopes 1,2 , and 3 .

The density at each subzone $c \in c(z)$ is $(28)$ evaluated at subzone center $\mathbf{x}_{c}$. The scale factor $f$ generalizes the subzonal density distribution. If $f=0$, the subzonal density is flat; if $f=-1$, we recover the density distribution in Test 1 ; and if $f>0$, the subzones are anti-correlated with the interzonal variation. Note that (4) is satisfied provided each zone position is equal to the average of its subzone positions. Figure 4 illustrates this density distribution on the zones, nodes, and subzones of an $8 \times 8$ square mesh. We use $f=1$ to illustrate the subzonal field.

Figure 5 contains convergence curves for two choices of scale factor $f$ : a constant factor and a variable factor. Left is the constant scale factor $f=1$. Dashed black lines have reference slopes 1, 2, and 3. In this example, as $N$ increases, the relative variation within each zone decreases; that is, the subzonal density field becomes smoother under mesh refinement. The relative 

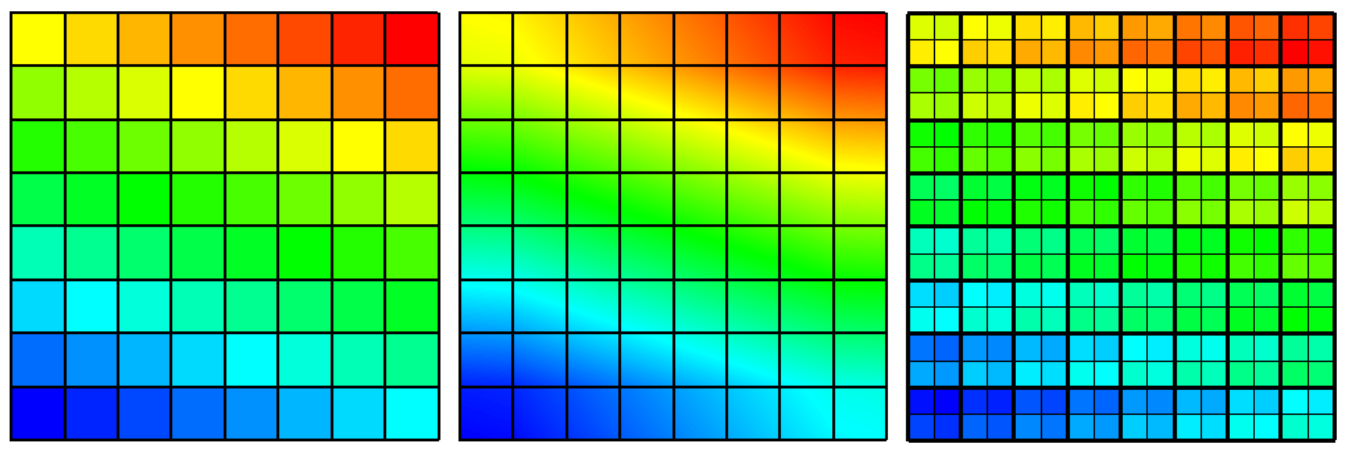

Figure 4: Initial discrete densities in zones (left), at nodes (center), and in subzones (right) for a linear field with anti- correlated subzonal variation.

errors and convergence rates of each reference match that in Test 1 . This test is again advantageous for the arithmetic average reference since it is exact in all interior subzones. Unlike Test 1, the zone density reference has lower absolute error than node density. This is because the discrete node densities are farther from the analytic profile at the boundary than the discrete zone densities when their values are initialized using (28).

The right plot contains a more challenging test for the reconstruction algorithm: a variable scale factor is chosen to increase with mesh resolution. The scale factor is as large as possible without having negative values in the initial discrete subzonal density field:

$$
f_{\max }=\frac{4 \rho_{0} N}{\|\nabla \rho\|_{1}},
$$

where $\|\nabla \rho\|$ is the 1 -norm of the density gradient in (27). The resulting subzonal field is discontinuous across zones despite the fact that the coarsened zonal and nodal fields are smooth. The dashed black line has slope 1. All references converge at first order, as expected for the discontinuous nature of the subzonal field. The zone density reference has minimal absolute error by nearly an order of magnitude. This is again due to the zone values being closer to the analytic profile at boundary subzones and thus committing a smaller error there. The steeper subzonal slope only exacerbates this effect. 

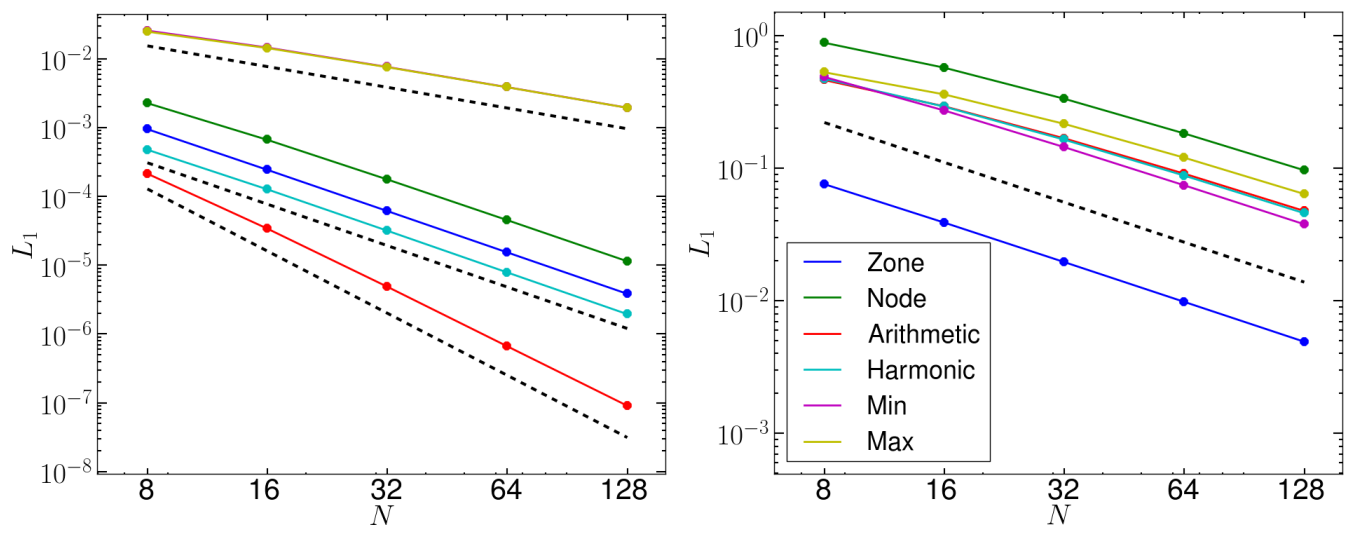

Figure 5: Null problem, linear field: $L_{1}$ convergence in subzonal density. Scale factor $f=1$ with reference slopes 1,2 , and 3 (left). Maximum scale factor $f_{\max }$ with reference slope 1 (right).

\section{Test 3: Discontinuous Density Field, Zero Subzonal Variation}

We consider a discontinuous density profile. Direction vector $\mathbf{r}$ points perpendicular to a linear discontinuity passing through point $\mathbf{c}$ and separating high and low density values $\rho_{H}$ and $\rho_{L}$. The initial density profile is

$$
\rho(\mathbf{x})=\left\{\begin{array}{ll}
\rho_{H} & , \mathbf{r} \cdot(\mathbf{x}-\mathbf{c})<0 \\
\rho_{L} & , \mathbf{r} \cdot(\mathbf{x}-\mathbf{c}) \geq 0
\end{array} .\right.
$$

We first initialize the discrete density profile so there is no subzonal variation. The discrete zone density $\rho_{z}$ is computed by evaluating (29) at zone center $\mathbf{x}_{z}$. The discrete density in each subzone is equal to its associated zone density: $\rho_{c}=\rho_{z_{c}}$. For all subsequent examples, we set $\mathbf{r}=(4 / 3,1), \mathbf{c}=(1 / 2,1 / 2)$, and $\rho_{H}=1$. We vary $\rho_{L}$ to test the robustness of each reference to large density jumps.

Figure 6 contains convergence curves for the $L_{1}$ error in subzonal density for two choices of $\rho_{L}: 10^{-1}$ and $10^{-6}$. The black dashed lines have reference slope of 1 . The error curve for zone density is not provided because the reconstructed subzonal densities match their initial values to machine precision. This is to be expected for this particular test: the zone density reference automatically satisfies mass conservation constraints at all zones and nodes. Here, and in subsequent plots, solid markers indicate reconstructed densities 
that maintain positivity; ' $\mathrm{X}$ ' markers indicate negative reconstructed values that have been repaired according to the nonconservative local procedure. It is worth mentioning that local repair produces an error in a very small set of subzones and is not a significant contribution to the global $L_{1}$ error.

The node density and local maximum references fail to preserve positivity for both density jumps and carry the largest absolute errors. The arithmetic average reference maintains positivity for smaller density jump but fails when the discontinuity is more extreme. The local minimum and harmonic average references are robust to computing negative states (even in an extreme case of $\rho_{L}=10^{-12}$, not included.) The harmonic average outperforms the local minimum in all cases due to it being a smooth function. First order convergence is established for smaller density jumps. For the larger jump, the harmonic average and local minimum do not converge: errors are only committed at subzones along the discontinuity. The size of these errors and the number of subzones scale with the mesh resolution. Regardless, the errors are several orders of magnitude smaller than those committed by the other reference states.
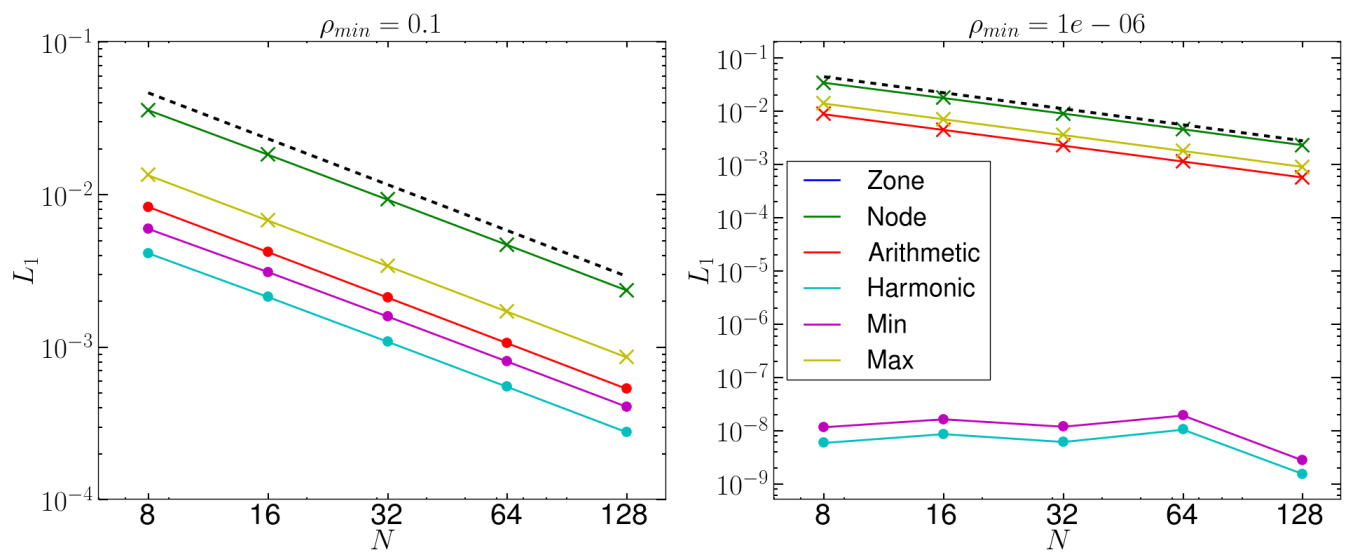

Figure 6: Null problem, discontinuous field: $L_{1}$ convergence in subzonal density for density jump of 10 (left) and $10^{6}$ (right). No variation in the initial subzonal density. Positive reconstructions have solid markers, repaired negative reconstructions have X. Dashed reference has slope 1. 


\subsubsection{Reconstructing a Linear Density Distribution}

We next test how the reconstruction performs when remapping onto a target mesh with different topology and geometry. We begin with the linear density distribution (27), again with $\rho_{0}=10$ and $\nabla \rho=(1,3)$, on a square $N \times N$ mesh. The subzonal variation follows the same distribution as the global density (i.e. equation (28) with $f=-1$ ).

We consider two target meshes: an unstructured mesh of arbitrary polygons and a structured mesh of high-aspect-ratio quads. The unstructured mesh consists of a random Voronoi tessellation having $N^{2}$ number of zones. The structured mesh consists of a $r N \times\left\lfloor\frac{N}{r}\right\rfloor$ lattice of rectangles, with $r>1$. For all subsequent tests, we use $N \in\{8,16,32,64,128\}$ and $r=4$. Figure 7 illustrates the zonal density on the initial mesh as well as its remapped values on the unstructured Voronoi mesh and high-aspect quad mesh.

Figure 8 gives $L_{1}$ convergence results for the reconstructed subzone densities relative to their analytic values from (27). For the Voronoi mesh (left), all reference values demonstrate first-order convergence. Arithmetic and harmonic averages are identical and outperform the other references on an arbitrary polygonal grid. In the high-aspect quad case (right), all smooth references converge at second order and non-smooth references (local minimum and maximum) at first order. The regularity of the high-aspect quad zones allows for second-order convergence in the reconstructed subzonal densities; second-order convergence on a polygonal mesh can be achieved provided greater regularity and mesh quality-for instance, a centroidal Voronoi tessellation composed of regular hexagons.

\subsubsection{Reconstructing a Density Discontinuity}

We repeat the above test for a density discontinuity of size 10 . We initialize the density fields in the same manner as Test 4 for the null problem tests. Here $\rho_{L}=10^{-1}$.

We again map onto an unstructured random Voronoi tessellation and a structured grid of high-aspect quads. Figure 9 illustrates this in zone density. Figure 10 gives convergence results for each reference state. Again, solid markers indicate positive reconstructed densities, and ' $\mathrm{X}$ ' markers indicate locally repaired negative densities. Because of the modest density jump and relative grid regularity, all references produce positive reconstructions for the high-aspect quad case. Only the zone density and local maximum references compute negative subzonal densities for the random Voronoi case. This is due to significant grid irregularity: the existence of small edges in the Voronoi 

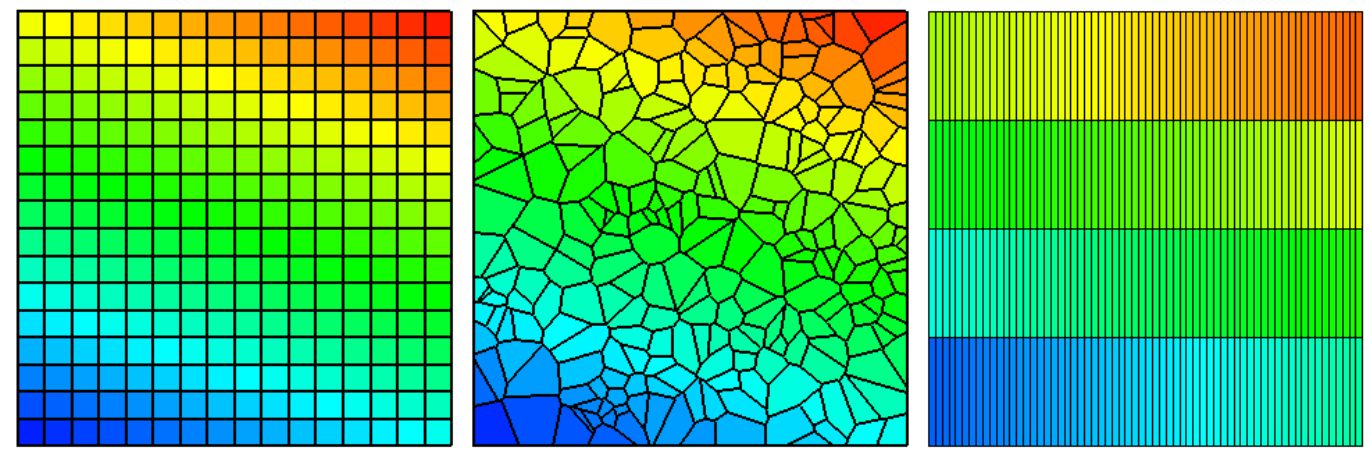

Figure 7: Initial linear zonal density (left) and remapped zonal density on unstructured polygons (center) and high- aspect-ratio quads (right).
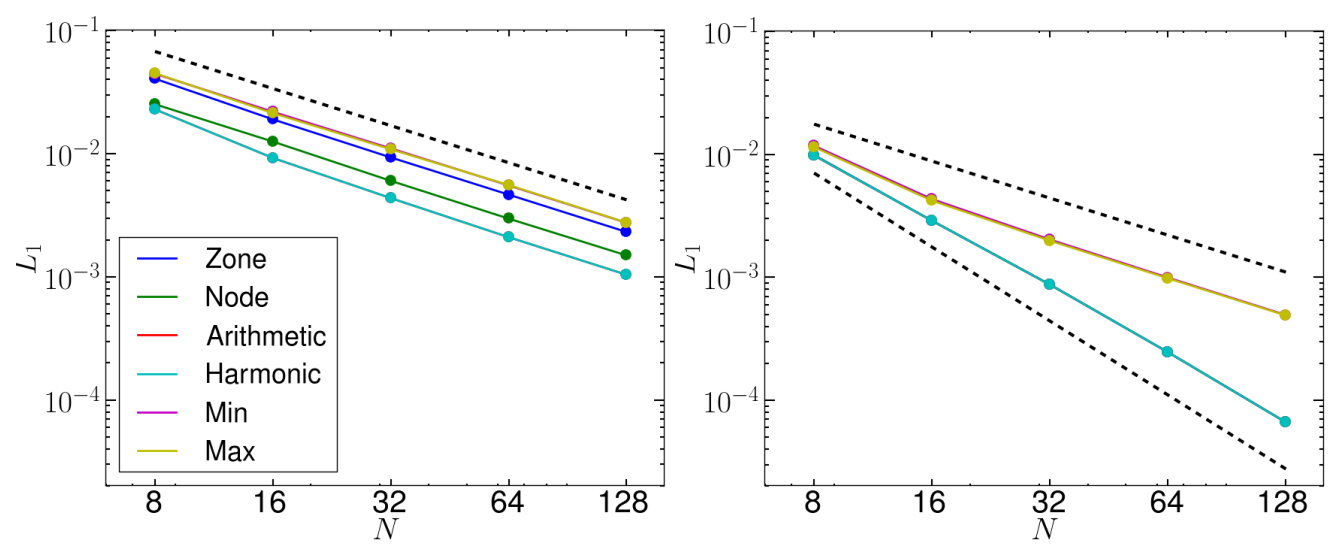

Figure 8: Reconstruct linear density field: $L_{1}$ convergence in subzonal density. Unstructured polygonal target mesh with reference slope 1 (left) and high-aspect-ratio target mesh with reference slopes 1 and 2 (right).

mesh leads to disparate subzonal volumes. Negative reconstructed values tend to occur in or near zones having small edges. Improving the quality of the grid, for instance by deleting or expanding small edges in the mesh, reduces negative reconstructions.

We also measure how well monotonicity is preserved in the reconstructed 

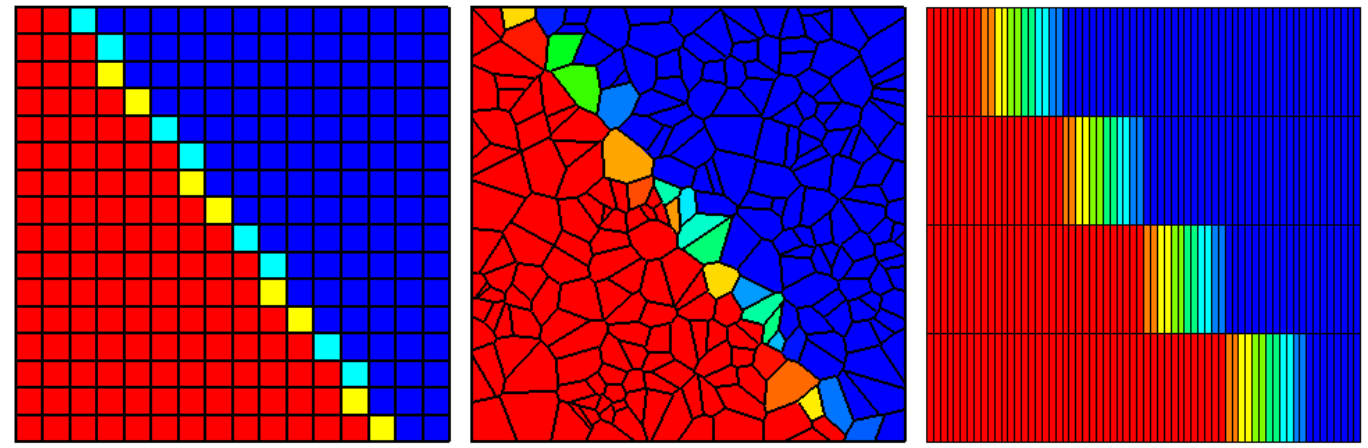

Figure 9: Initial discontinuous zonal density (left) and remapped zonal density on unstructured polygons (center) and high-aspect-ratio quads (right).
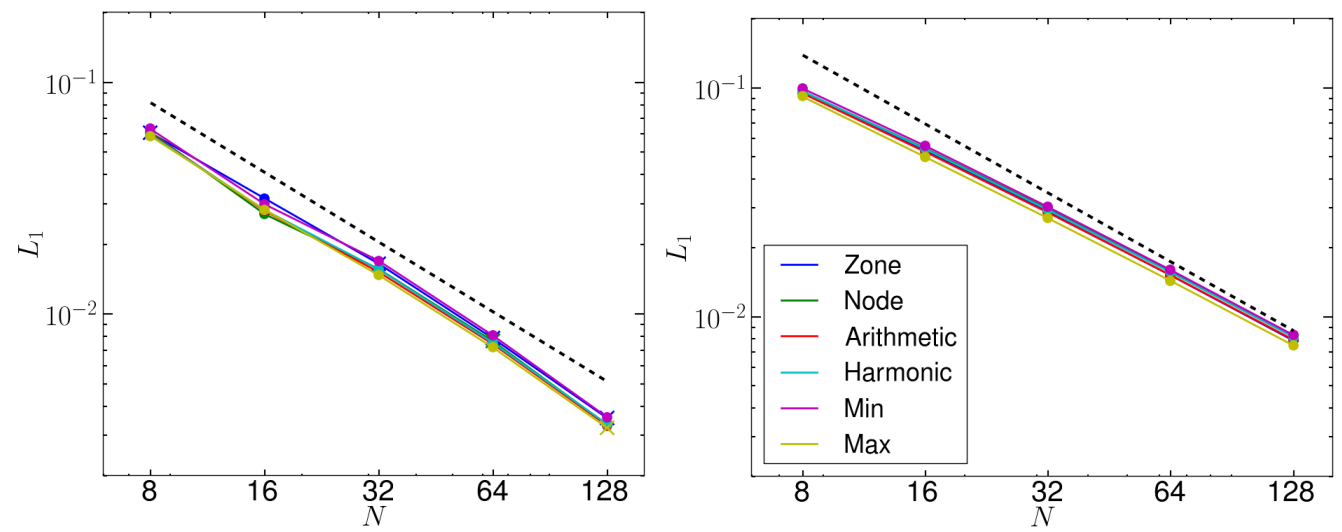

Figure 10: Reconstruct discontinuous density field: $L_{1}$ convergence in subzonal density. Unstructured polygonal target mesh (left) and high-aspect-ratio target mesh (right), each with reference slopes of 1 . Positive reconstructions have solid markers, repaired negative reconstructions have $\mathrm{X}$.

subzonal density field. For each test, we compute the ratio

$$
r_{\text {mono }}=\min _{c} \rho_{c} / \max _{c} \rho_{c} .
$$

If the reconstruction is monotone, $r_{\text {mono }}$ will be 0.1 for this problem. Ratio values are provided in table 3 and table 4 for the unstructured Voronoi and high-aspect quad target meshes respectively. Empty entries correspond to 
cases of negative reconstructed densities. In all instances, the reconstructed values are non-monotonic, with more extreme ratio values occurring for the unstructured Voronoi target mesh. It is worth reiterating that the monotonicity being measured here is in the subzonal densities: the directly remapped zonal and nodal densities maintain monotonicity exactly. Although the subzonal densities do not respect the monotonicity limits of their coarsened nodal and zonal values, they do rigorously reproduce the remapped zonal and nodal mass densities due to the constraints imposed. This is adequate in the context of the staggered hydrodynamic method towards which reconstruction is applied; it is the compromise we accept in order to allow the significant computational savings compared with directly remapping subzonal quantities.

From the data collected, the zone density, node density, and local maximum reference states appear unsatisfactory due to both the size of monotonicity errors and their tendency to compute negative values. The local minimum reference is most robust to computing negative states; however, it is a discontinuous function of local data and has poor convergence properties for smooth fields as a result. The arithmetic and harmonic averages have similar convergence properties, but the latter is more robust to computing negative states. The remaining tests use the harmonic average reference for this reason. Additional reference comparisons are included to aid discussion, but the harmonic average is our choice moving forward.

\begin{tabular}{ccccccc}
\hline$N$ & Zone & Node & Arith. Avg. & Harm. Avg. & Local Min & Local Max \\
\hline 8 & & 0.0266 & 0.0436 & 0.0566 & 0.0667 & 0.0455 \\
16 & 0.0027 & 0.0716 & 0.0583 & 0.0671 & 0.0560 & 0.0453 \\
32 & & 0.0331 & 0.0198 & 0.0281 & 0.0295 & 0.0176 \\
64 & & & 0.0354 & 0.0350 & 0.0425 & 0.0222 \\
128 & & 0.0129 & 0.0260 & 0.0359 & 0.0342 & \\
\hline
\end{tabular}

Table 3: Reconstruct discontinuous density field: monotonicity metric convergence for unstructured polygonal target mesh. (Monotone reconstruction has metric value 0.1.) Missing entries indicate cases that failed due to negative reconstructed densities.

\subsubsection{Cyclic Remap Tests}

This is a remapping test based on the work of [12] and used in [21] to test flux-based ALE remap strategies. The initial mesh configuration consists of a 


\begin{tabular}{ccccccc}
\hline$N$ & Zone & Node & Arith. Avg. & Harm. Avg. & Local Min & Local Max \\
\hline 8 & 0.0547 & 0.0149 & 0.0646 & 0.0665 & 0.0782 & 0.0565 \\
16 & 0.0530 & 0.0141 & 0.0651 & 0.0663 & 0.0782 & 0.0572 \\
32 & 0.0524 & 0.0142 & 0.0654 & 0.0663 & 0.0782 & 0.0575 \\
64 & 0.0522 & 0.0143 & 0.0655 & 0.0664 & 0.0783 & 0.0577 \\
128 & 0.0522 & 0.0143 & 0.0656 & 0.0664 & 0.0783 & 0.0578 \\
\hline
\end{tabular}

Table 4: Reconstruct discontinuous density field: monotonicity metric convergence for high-aspect quad target mesh. (Monotone reconstruction has metric value 0.1.)

lattice of quads. Mesh nodes are iteratively moved according to the following periodic displacement:

$$
\begin{aligned}
& x_{k}\left(x_{0}, y_{0}, \tau\right)=x_{0}+F(\tau) \sin \left(2 \pi \tau x_{0}\right) \sin \left(2 \pi \tau y_{0}\right) \\
& y_{k}\left(x_{0}, y_{0}, \tau\right)=y_{0}+F(\tau) \sin \left(2 \pi \tau x_{0}\right) \sin \left(2 \pi \tau y_{0}\right) \\
& F(\tau)= \begin{cases}0.2 \tau & , \quad \tau \in[0,0.5] \\
0.2(1-\tau) & , \quad \tau \in[0.5,1]\end{cases} \\
& \tau=k / k_{\max }
\end{aligned}
$$

Here $k$ is the remap iteration, $\left(x_{0}, y_{0}\right)$ is the initial position of each node, $\left(x_{k}, y_{k}\right)$ is the $k$-th position, and $\tau$ is the pseudo-time period of the displacement. Mesh nodes return to their original positions after $k_{\max }$ total iterations.

Initial background density and velocity fields are iteratively overlaid onto the new displaced mesh. We consider two initial fields: one smooth and one discontinuous.

\section{Smooth Field}

We consider smooth profiles for density and velocity

$$
\begin{aligned}
\rho(x, y) & =\rho_{\text {back }}+\rho_{a m p} \sin (2 \pi x) \sin (2 \pi y) \\
u(x, y) & =\frac{x}{\sqrt{x^{2}+y^{2}}} V_{a m p} \sin (2 \pi x) \sin (2 \pi y) \\
v(x, y) & =\frac{y}{\sqrt{x^{2}+y^{2}}} V_{a m p} \sin (2 \pi x) \sin (2 \pi y)
\end{aligned}
$$


Figure 11 illustrates these profiles for $\rho_{b a c k}=\rho_{a m p}=1$ and velocity amplitude $V_{a m p}=1$.
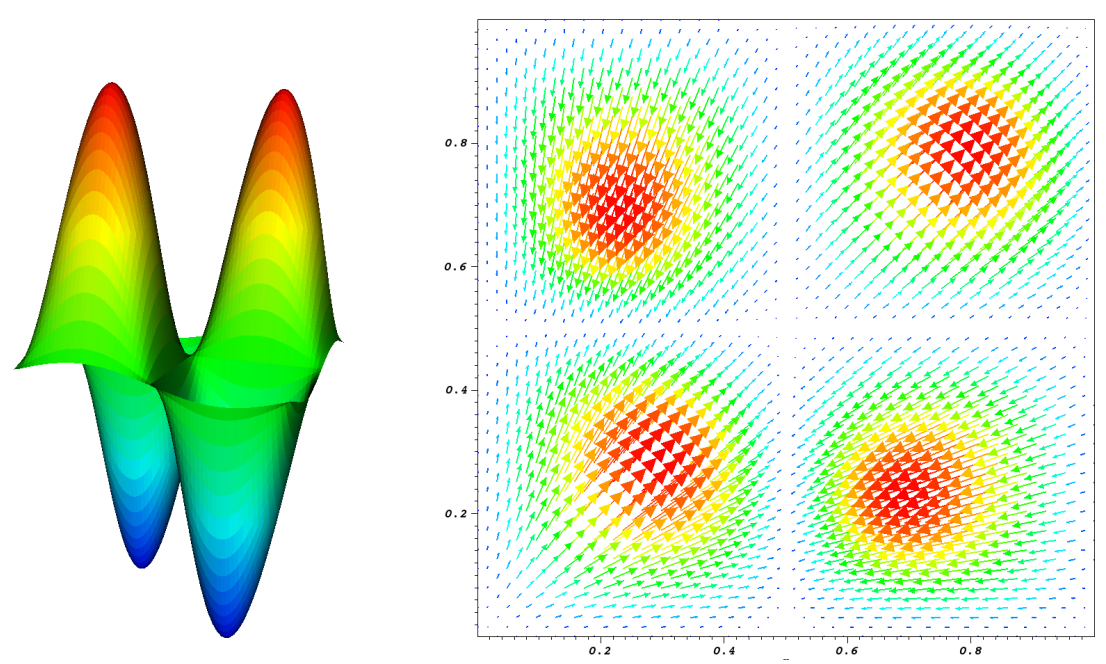

Figure 11: Surface plot of the initial smooth density field (left) and vector plot of the initial smooth velocity field (right).

Differences in accuracy among the various reference states is negligible due to the smoothness of the underlying profiles. Figure 12 reports $L_{1}$ convergence results for the remapped zonal density, nodal density, and radial velocity for the harmonic average reference state only. Results correspond to an $N \times N$ square mesh with $N \in\{32,64,128,256,512\}$. The overlay remap package is formally second-order accurate. Our reported convergence rates converge the order of accuracy of the overlay: 2.04, 1.97, and 2.12 for zonal density, nodal density, and radial velocity respectively.

\section{Discontinuous Field}

We consider two discontinuous regions of the mesh: a high-density cylindrical shell, having $\rho_{\text {shell }}=1$ and unit, outward-pointing velocity $\mathbf{u}_{\text {shell }}=\hat{\mathbf{x}}_{r}$ and a low-density background having $\rho_{\text {background }}=1 / \mathrm{J}$ and zero velocity. Here $\mathbf{x}_{r}=\mathbf{x}-(0.5,0.5)$ defines a radial position and $J>1$ defines a jump in density. The shell has inner and outer radii $r_{\text {inner }}=0.25$ and $r_{\text {outer }}=0.45$, centered on the unit square. Figure 13 gives the initial density (zonal and subzonal) and nodal velocity magnitude. Density is initialized to have zero subzonal variation. 

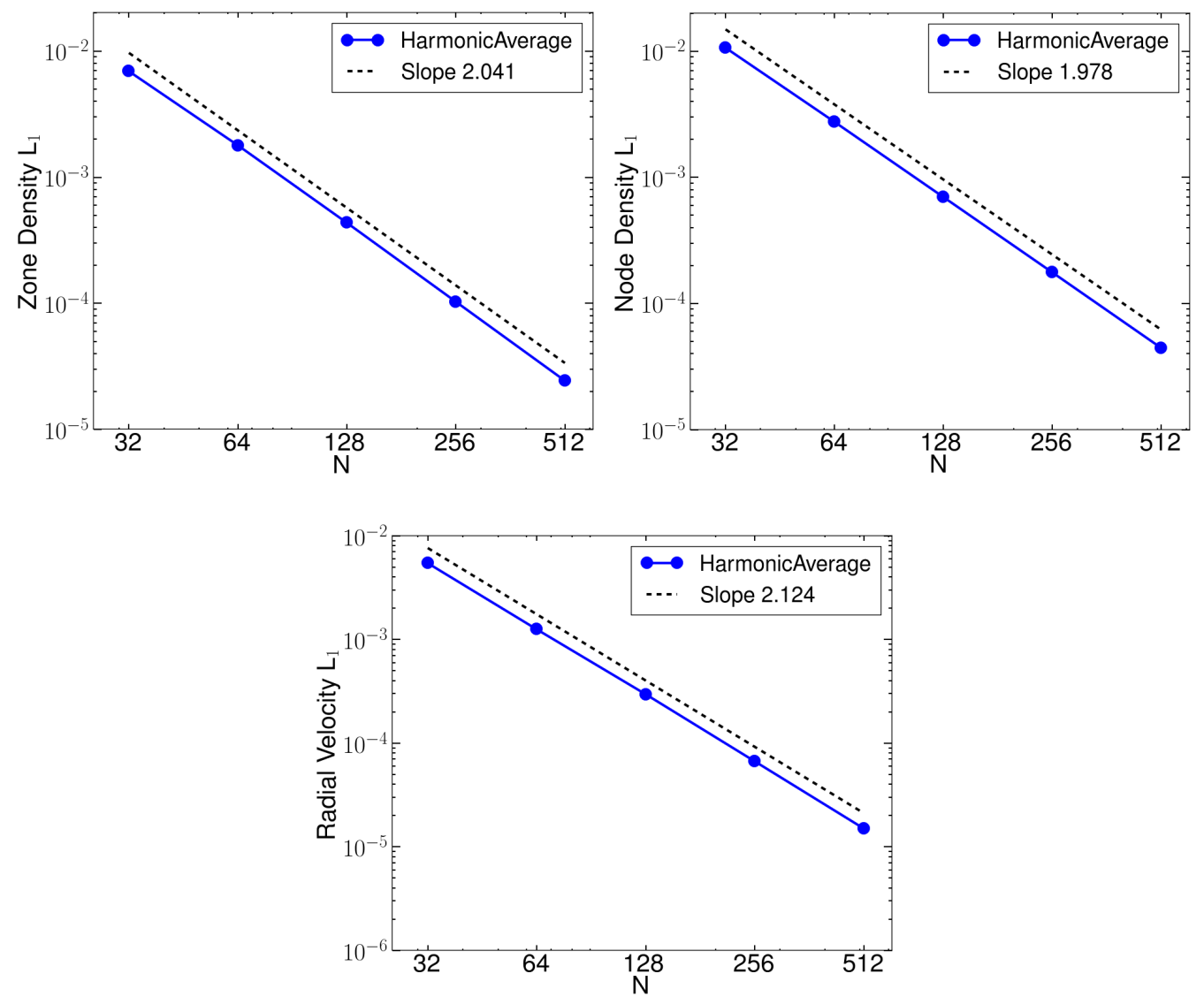

Figure 12: Cyclic remap, smooth fields: $L_{1}$ convergence in remapped zonal density, nodal density, and radial velocity with linear fit.

Figure 14 illustrates the reconstructed subzonal density and nodal velocity magnitude at the time of maximum distortion $(\tau=1 / 2)$ and at the final time $(\tau=1)$. Velocity is an important accuracy metric for a staggered-grid remap due to the fact that it is not independently remapped. Instead, the nodecentered momentum density $\mathbf{p}_{n}=\rho_{n} \mathbf{u}_{n}$ is remapped, with velocity recovered through division of the post-remap momentum density by the post-remap nodal mass density.

If subzonal densities do not satisfy (5) exactly, then the recovered postremap velocity will not satisfy linear momentum conservation. For this prob- 

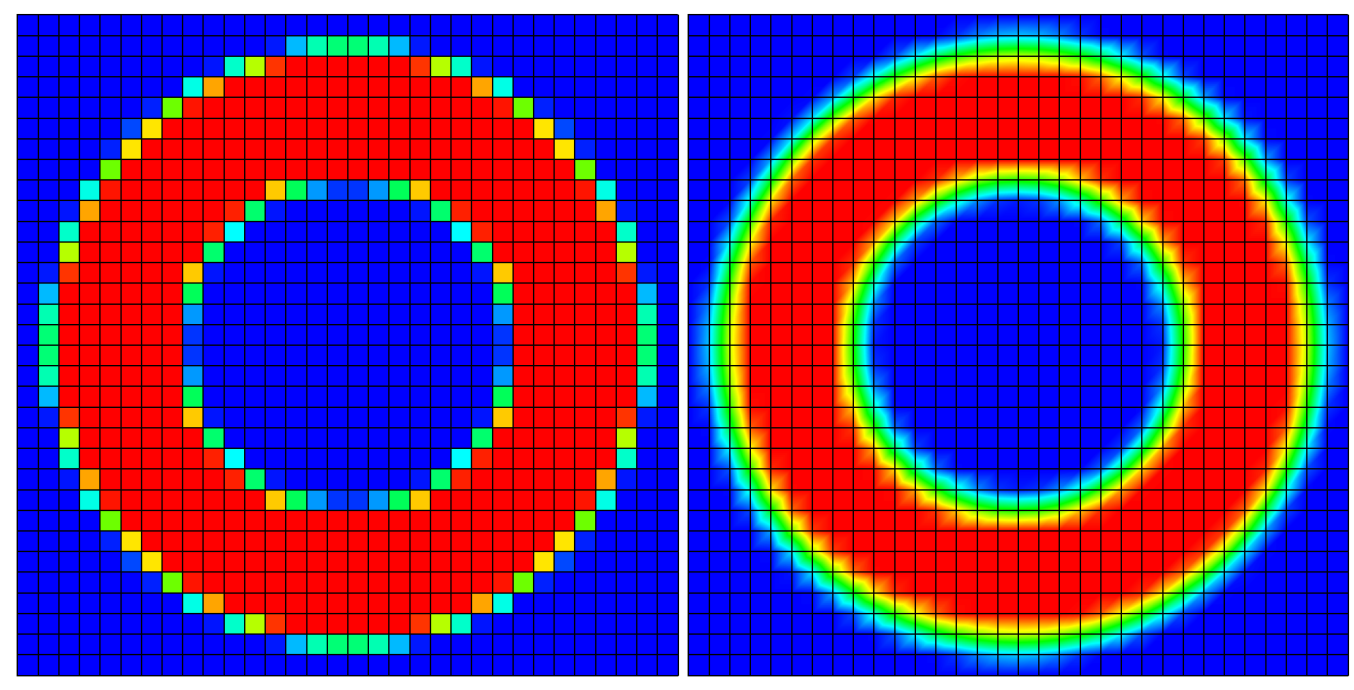

Figure 13: Initial density (left) and velocity magnitude (right) for the discontinuous cyclic remap test. The density in each subzone is initialized to be equal to its zone density.

lem, the total linear momentum is zero by construction. The harmonic average reference computes positive reconstructed densities for both $J=10$ and $J=1000$ and therefore conserves linear momentum to roundoff. Momentum conservation errors are observed for the other reference states when $J=1000$ due to our repairing negative reconstructed values.

The velocity direction is also preserved during the remap. We define a direction error per node $E_{n}^{\text {dir }}$ using the following formula:

$$
E_{n}^{d i r}=\left|\frac{\mathbf{u}_{n}}{\left\|\mathbf{u}_{n}\right\|} \cdot \widehat{\mathbf{r}}_{n}-1\right|\left\|\mathbf{u}_{n}\right\|
$$

where $\mathbf{u}_{n}$ is the computed velocity and $\widehat{\mathbf{r}}$ is the radial direction vector. The error is scaled by the norm of the velocity to ensure velocity values close to zero do not significantly contribute. Figure 15 gives the distribution of this error for $J=1000$ at both $\tau=1 / 2$ and $\tau=1$.

Additionally, fig. 16 compares the pseudo-time history of the ratio of minimum to maximum density (30) for both $J=10$ and $J=1000$ using the harmonic average reference. For nodal and zonal density, (30) is constant, implying monotonicity is preserved in each quantity. We have not imposed explicit monotonicity constraints on the reconstructed subzonal densities; as 

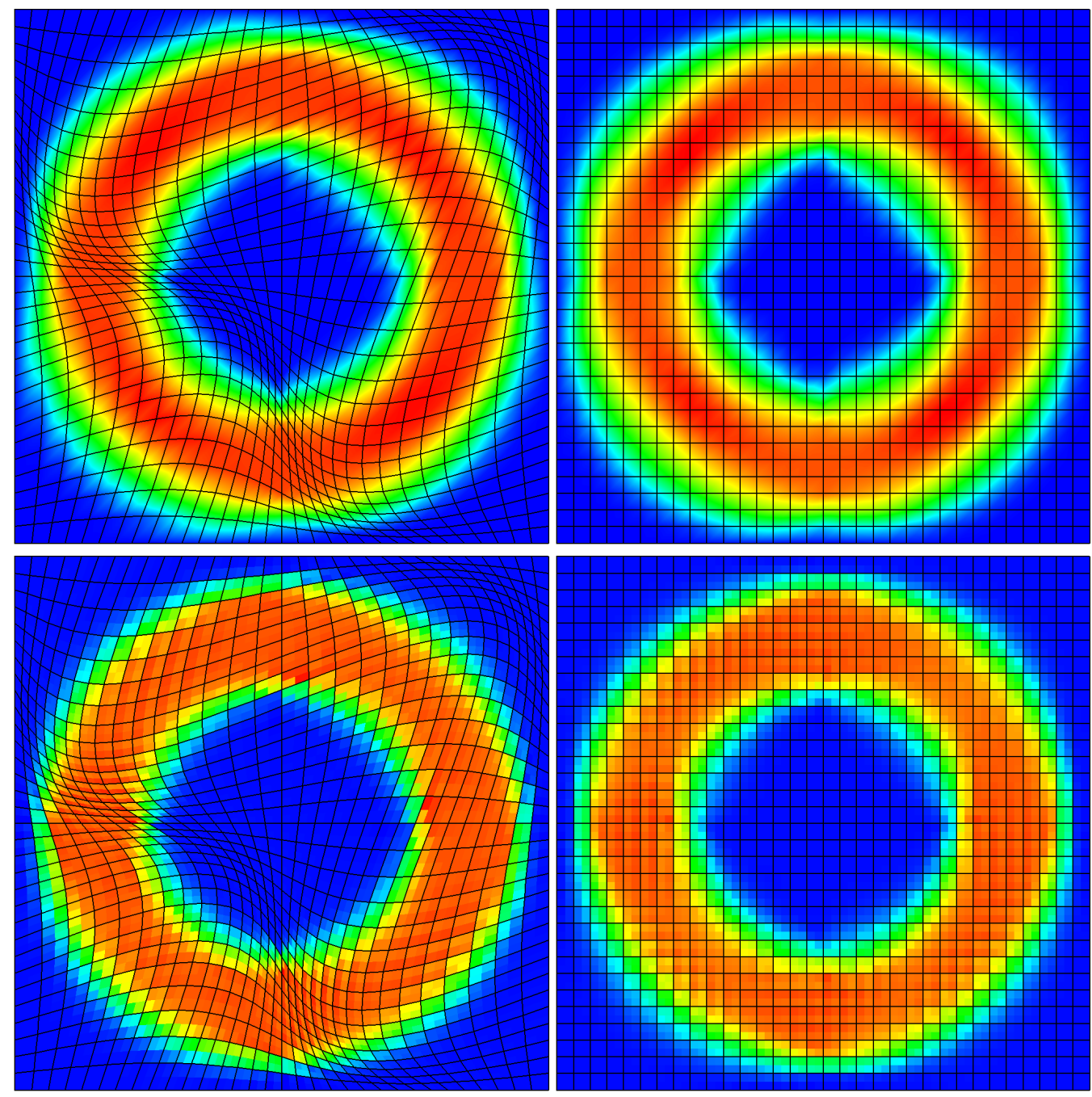

Figure 14: Cyclic remap, discontinuous field: velocity magnitude (top row) and subzonal density (bottom row). Fields at maximum mesh distortion $(\tau=1 / 2$, left column) and after distortion is reversed ( $\tau=1$, right column). Subzonal density reconstruction with harmonic average reference was applied to make these plots.

such, this quantity is not constant in pseudo-time. (See appendix C for a discussion on imposing monotonicity constraints for the reconstruction procedure.) 

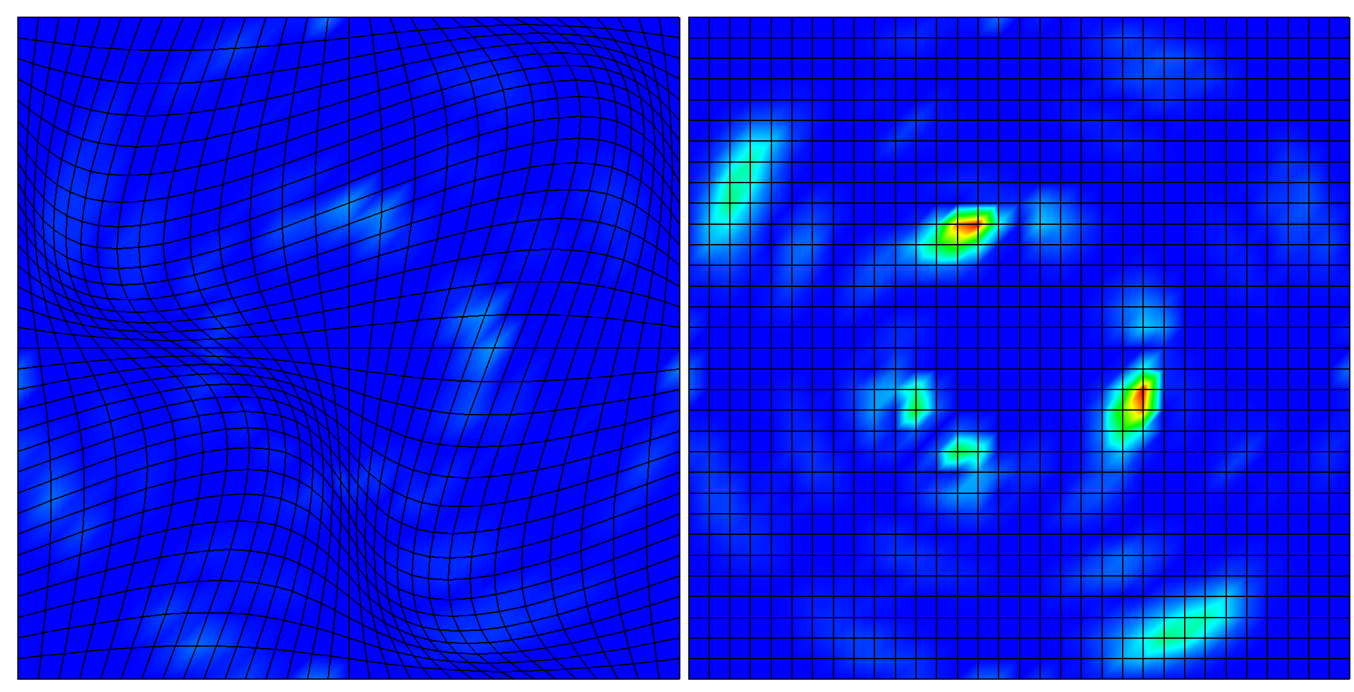

Figure 15: Cyclic remap, discontinuous field: error in velocity direction at each node at times of maximum mesh distortion $(\tau=1 / 2$, left $)$ and final time $(\tau=1$, right). The color map displays errors in the range $[0,0.007]$.
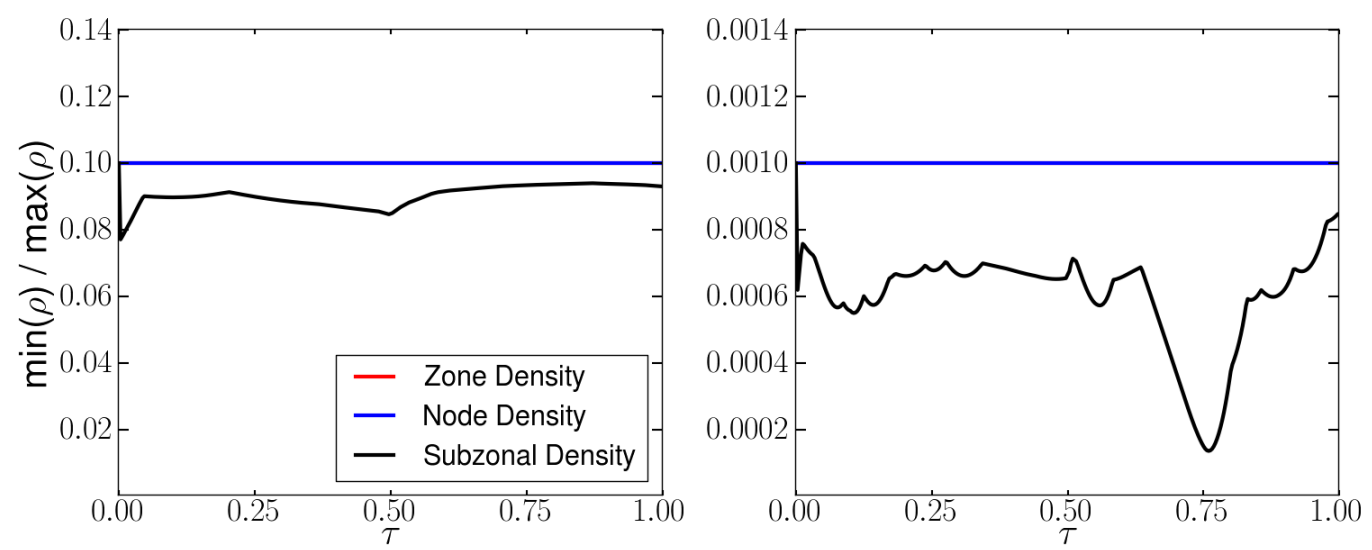

Figure 16: Cyclic remap, discontinuous field: monotonicity ratio as a function of pseudotime $\tau$ for initial density jumps $J=10$ (left) and $J=10^{3}$ (right). Remapped zone and node density do not contain new extrema and are constant in pseudo-time. Subzonal density computed using harmonic average reference.

Finally, fig. 17 gives $L_{1}$ convergence results for zonal density, nodal density, and radial velocity. The existence of discontinuities in density and ve- 
locity imply that the maximum possible convergence rate is one. We report rates of $0.66,0.54$, and 0.55 for zonal density, nodal density, and radial velocity respectively. This is consistent with results in [21] for a comparable flux-based ALE procedure.
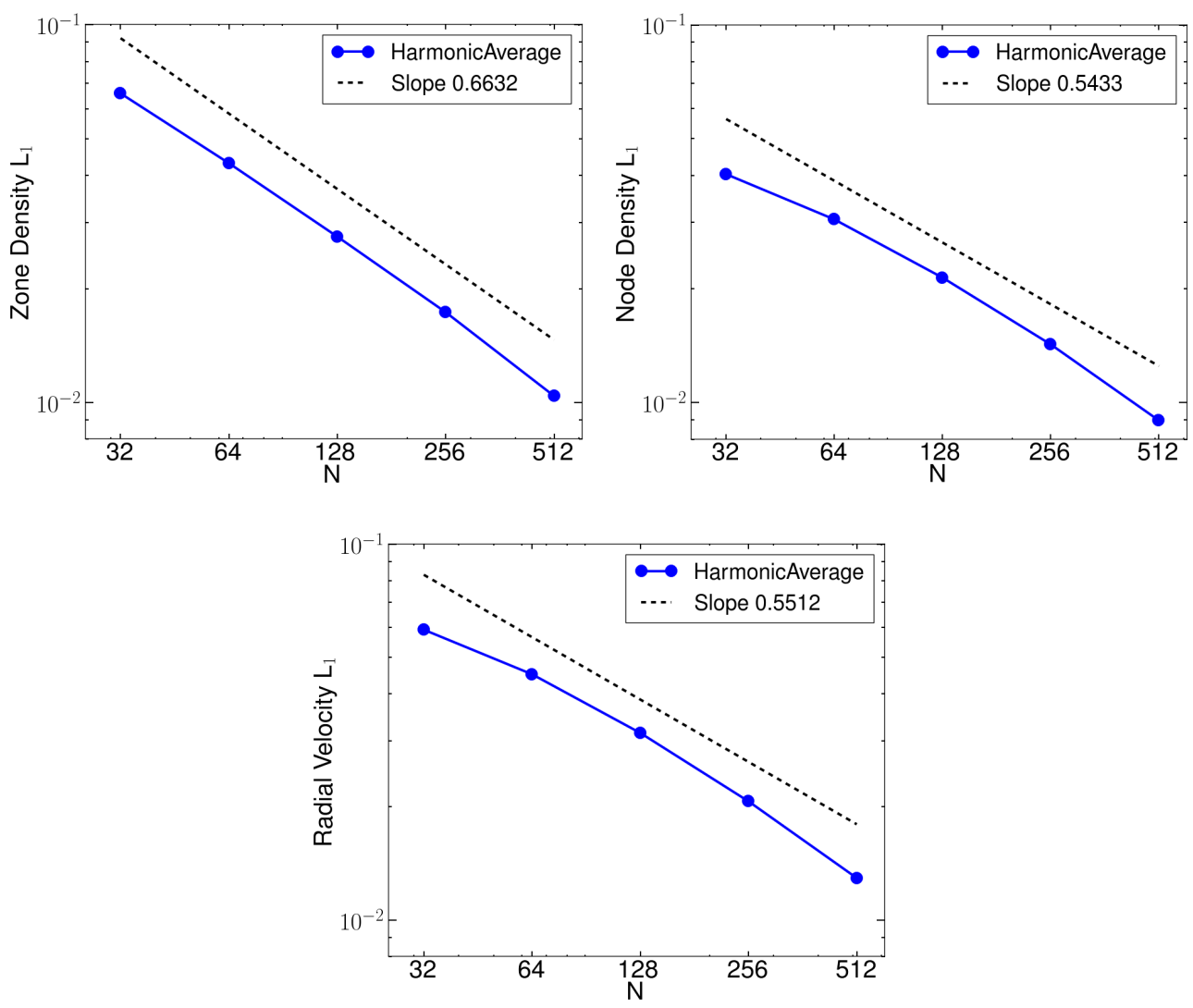

Figure 17: Cyclic remap, discontinuous fields: $L_{1}$ convergence in remapped zonal density, nodal density, and radial velocity with linear fit.

\subsection{Hydrodynamic Tests}

We compute hydrodynamics tests using the typical "Lagrange + Remap" methodology of ALE. Solution variables are updated using the staggered, compatible Lagrangian hydro discretization [2]. Mesh nodes move due to the Lagrangian-frame fluid velocity. At the end of each hydro step, we perform 
an ALE-based remap: all node positions are relaxed using one iteration of the Winslow-Crowley-based elliptic mesh smoothing algorithm [1]. Updated solution variables are then overlaid onto the relaxed mesh using Overlink [6]. The reconstruction algorithm completes the simulation cycle by generating conservative subzonal densities.

It's important to point out that the ALE strategy we employ-relaxing every mesh node at every cycle, overlaying the post-Lagrange state, then reconstructing subzonal densities - is not intended to be more numericallyaccurate. (In fact, for the problems we consider, a purely-Lagrangian solution maintains better mesh resolution and numerical accuracy.) The intention is to apply the new reconstruction algorithm every cycle and verify its convergence properties. The hydro tests we have selected have analytic solutions; our results may be compared with those in [21], which utilizes an identical hydro discretization.

\subsubsection{Sedov-Taylor Blast Wave}

We analyze the Sedov-Taylor blast wave [22, 23, 24]; this models the shock expansion of an initially pressureless, constant-density gas due to a high-energy point source. Exact similarity solutions exist in planar (1D), cylindrical (2D), and spherical (3D) geometries. We initialize the blast wave with a unit energy density spike in the center of a zero-pressure ideal gas having $\gamma=1.4$ and unit initial density. This yields a peak shock density of 6. Each simulation is advanced until the analytic solution predicts the shock position to be at a radial distance of 0.4 . We compute numerical solutions on two separate grids: a regular $N \times N$ square grid and a radially-symmetric unstructured grid of arbitrary polygons. In all test cases, the reconstruction algorithm preserves symmetry in the computed solution.

\section{D Blast Wave on a Regular Grid}

As an example of the necessity for a subzonal remap treatment that respects both the zonal and nodal remapped masses, we first compare the results of a flattened reconstruction to the new reconstruction algorithm with harmonic average reference state. Nodal density before and after a remap are inconsistent for the flattened reconstruction, and linear momentum is consequently not conserved. The effect is most apparent in pressure. Figure 18 compares the computed final pressure profile on a $128 \times 128$ square grid using the two reconstruction strategies. In the flattened reconstruction example, the shock front lags the analytic location, and the post-shock state 
is incorrect. The new reconstruction algorithm conserves linear momentum to round-off and correctly captures the analytic shock front.
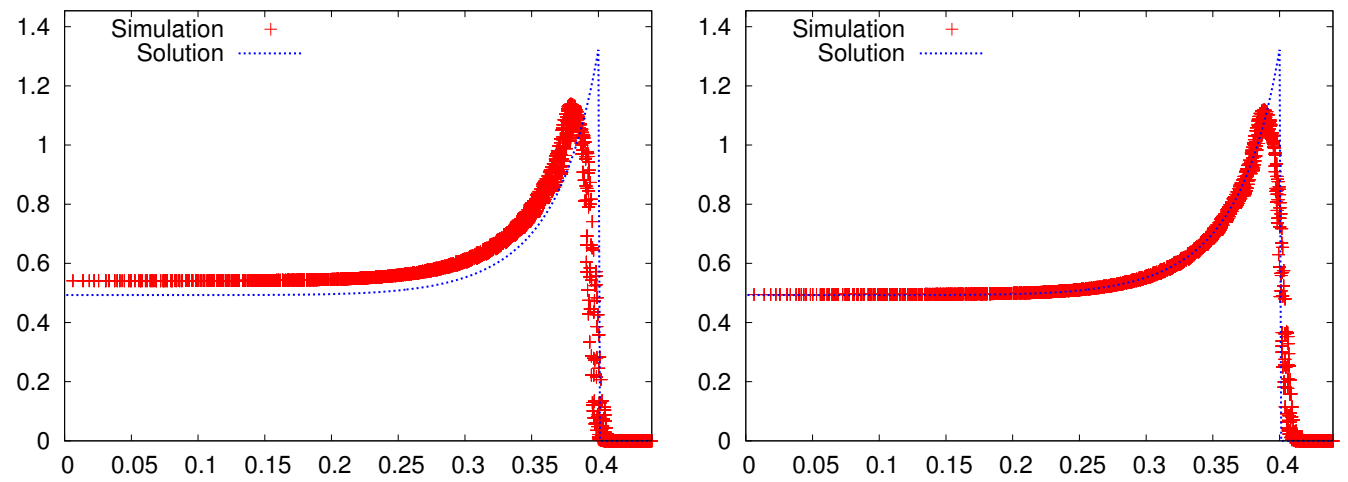

Figure 18: Sedov problem on $128 \times 128$ square mesh. Final pressure profile for flattened reconstruction of the subzonal densities (left) and a variation-diminishing reconstruction with harmonic average reference (right).

Figures 19 and 20 compare the zone density contours and radial profiles for the flattened and reconstructed calculations respectively. The solution using reconstruction captures the shock front more accurately and maintains a steeper peak density. Numerical diffusion at the shock front is to be expected and is due to our relaxing the mesh and overlaying the solution at every cycle. Our reasons for doing so are discussed above.

Figure 21 illustrates the reconstructed subzonal density and radial profile for this problem. The reconstructed density matches the same solution profile as fig. 20. However, monotonicity errors are apparent throughout the solution. Distribution of corner density within a zone is not constant; in some places, it is even misaligned with the interzonal density variation, with overshoots and undershoots visible across zone faces. The lack of strict monotonicity manifests as increased scatter in the radial plot. The Lagrange hydro is not sensitive to this effect since the coarsened zonal and nodal representations of density are each monotone. The radial zonal and nodal densities are overlaid in fig. 21 to better illustrate this.

Figure 22 compares convergence rates for flattened subzonal densities versus reconstructed values using harmonic average reference. $L_{1}$ error is evaluated between the computed density, radial velocity, and pressure and their analytic values at the same spatial locations. Results were computed 

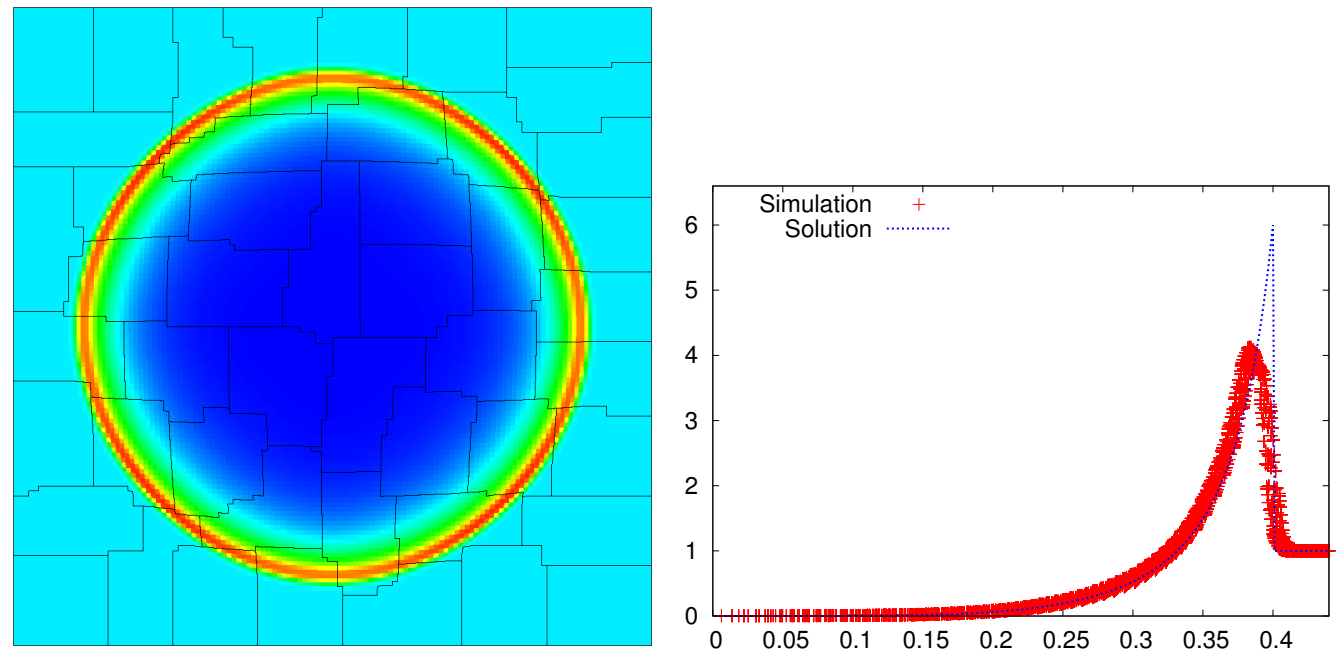

Figure 19: Sedov problem on $128 \times 128$ square mesh with nonconservative, flattened subzonal densities. Zone density color map with processor boundaries highlighted (left) and radial profile of zone density values (right).
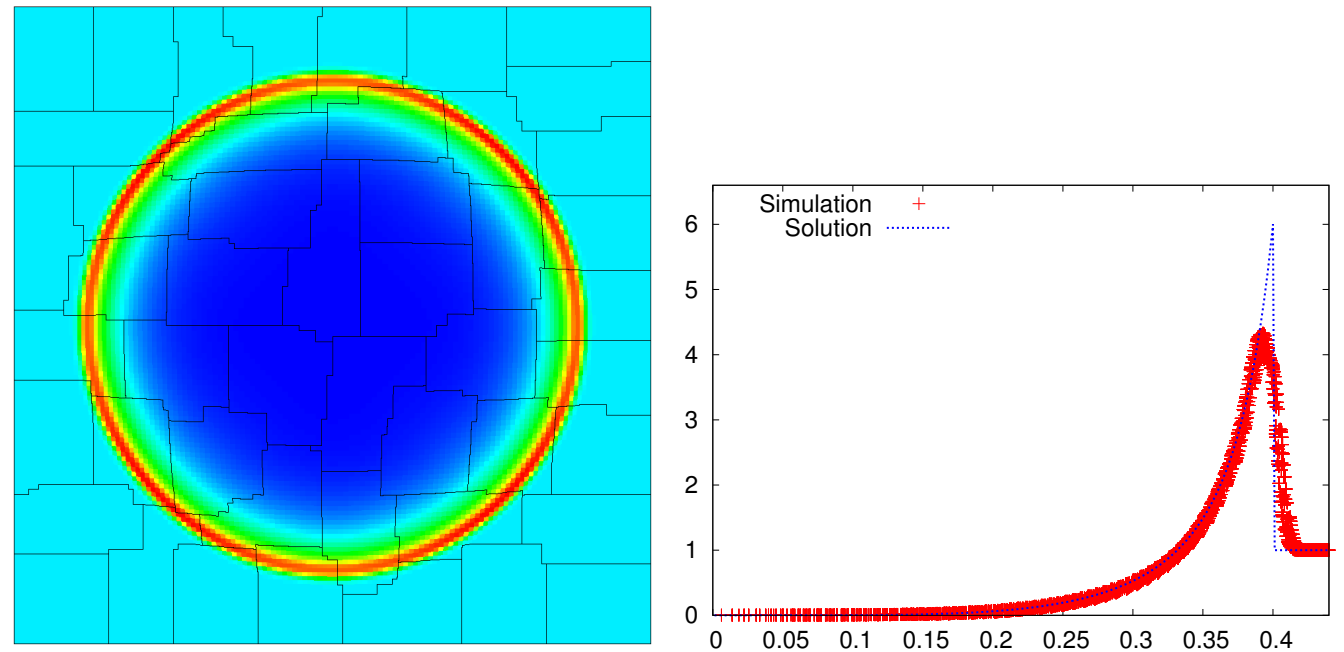

Figure 20: Sedov problem on $128 \times 128$ square mesh with conservative, reconstructed subzonal densities using harmonic average reference. Zone density color map with processor boundaries highlighted (left) and radial profile of zone density values (right). 

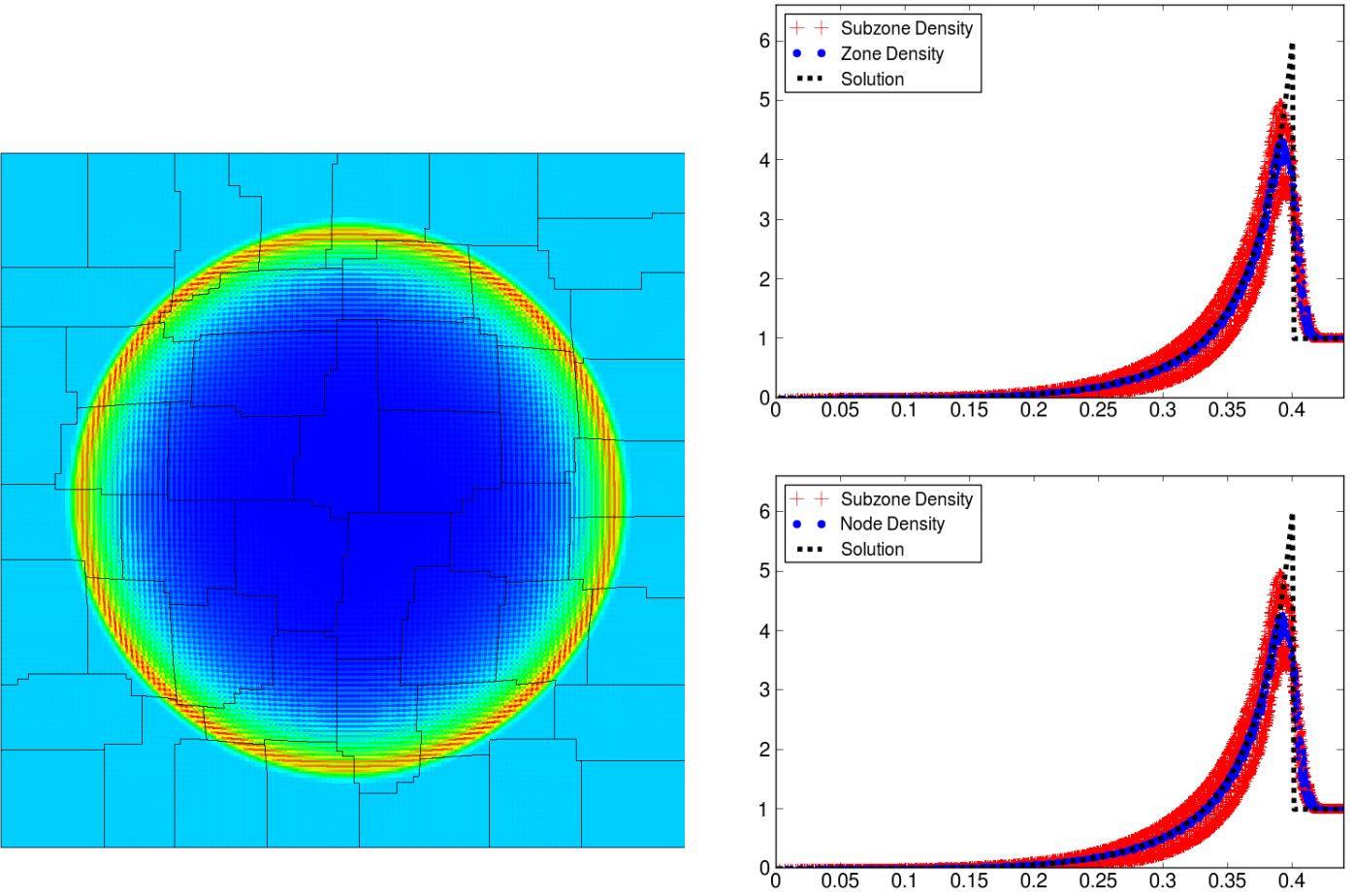

Figure 21: Sedov problem on $128 \times 128$ square mesh with conservative, reconstructed subzonal densities using harmonic average reference. Subzonal density color map with processor boundaries highlighted (left). Radial profile of subzonal density values in red (right) with corresponding zone densities (top right) and node densities (bottom right) in blue.

on an $N \times N$ square mesh for $N \in\{32,64,128,256,512\}$. Conservation errors cause the flattened reconstruction to diverge under mesh refinement, as expected for a shock-dominated test problem such as this. The presence of a strong, symmetric shock front and a relatively-stagnant post-shock state makes the Sedov blast wave a notoriously difficult problem to demonstrate optimal first-order convergence in most hydro schemes. We report converge rates of $0.72,0.78$, and 0.83 in density, radial velocity, and pressure respectively. Results are consistent with those obtained in [21] using the same Lagrangian hydro solver. 

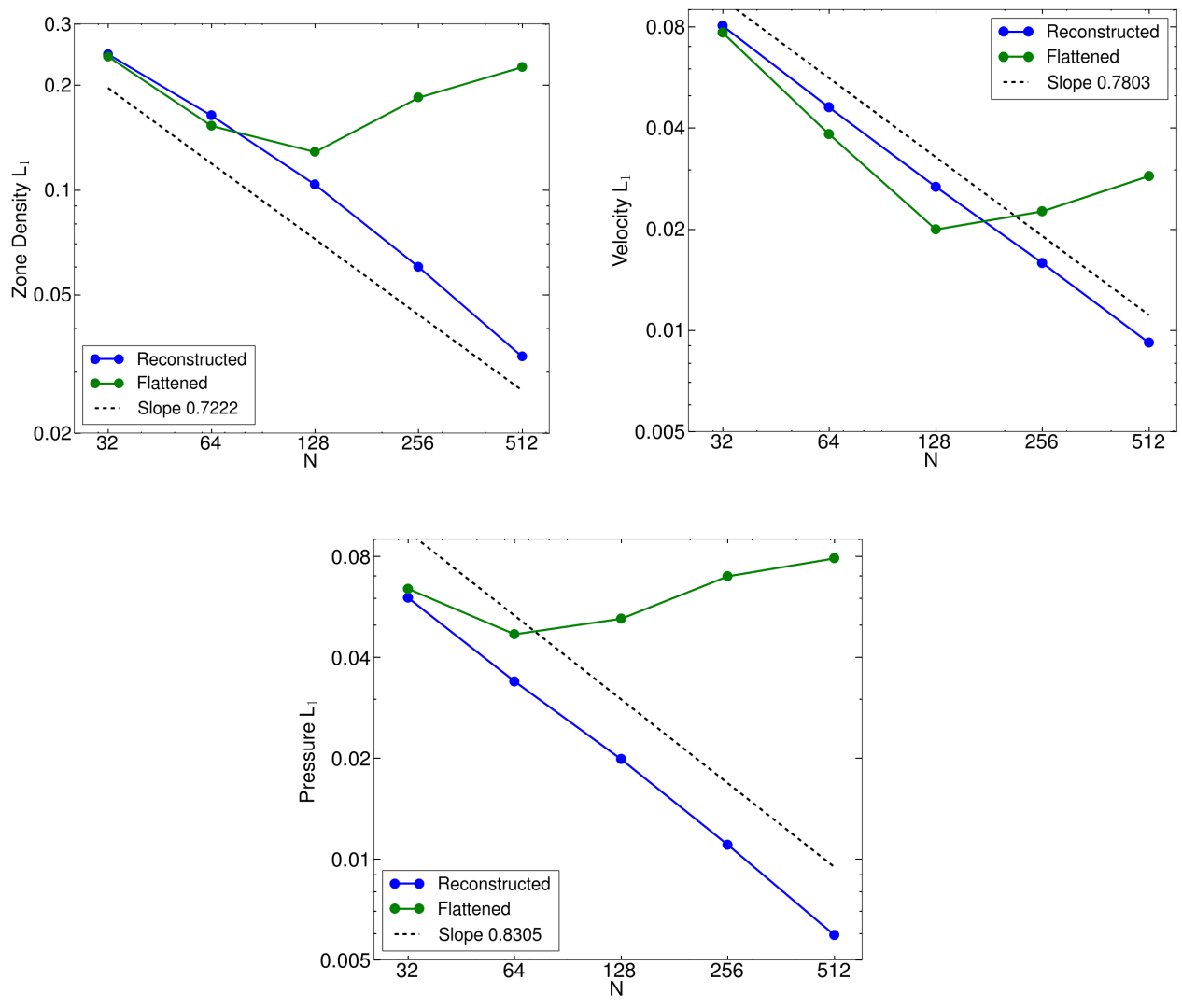

Figure 22: Sedov problem on a structured quad mesh. Plotted are $L_{1}$ convergence in zone density, radial velocity, and pressure comparing a nonconservative, flattened reconstruction to a conservative reconstruction with harmonic average reference.

\section{D Blast Wave on an Unstructured Polygonal Grid}

We repeat the above calculation on a grid composed of arbitrary polygons. We employ the same Voronoi gridding strategy as [21]: the initial mesh consists of a Voronoi tessellation constructed from regularly-spaced rings of generating points. For $N_{r}$ radial rings, we have a radial grid spacing of $1 / N_{r}$ between rings. Generating points are distributed on each ring so that the circumferential spacing matches the radial grid spacing as best as possible. We consider meshes corresponding to $N_{r} \in\{16,32,64\}$.

Figures 23 and 24 compare the zone density contours and radial pro- 
files for the flattened and reconstructed calculations respectively. Again, the reconstructed solution attains a steeper peak density and more-accurately captures the shock front. The effect is more exaggerated on an unstructured polygonal grid compared to the structured quad results. The reconstructed subzonal densities are illustrated in fig. 25. As before, the coarsened zonal and nodal density fields are overlaid onto the radial plot to illustrate that zonal and nodal density remain monotone even though the reconstructed values are not.
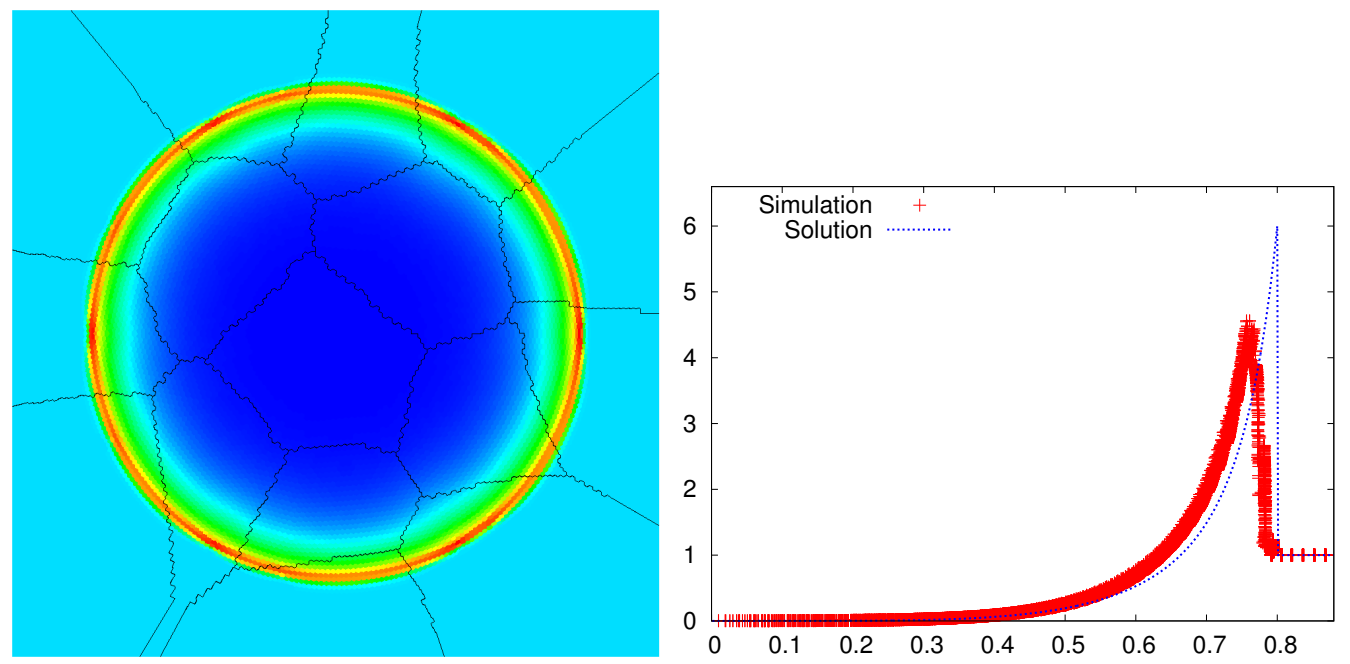

Figure 23: Sedov problem on radial Voronoi mesh with $N_{r}=64$. Zonal density using flattened reconstruction.

Figure 26 compares convergence rates for the flattened and variationdiminishing reconstruction. As with the structured mesh calculation, the flattened solution diverges under mesh refinement due to conservation errors. We report convergence rates of $0.58,0.82$, and 0.64 for density, radial velocity, and pressure respectively for the reconstructed solution. Results are again comparable to those in [21]

\subsubsection{Noh Problem}

We analyze the cylindrically-symmetric Noh test problem [20], modeling the implosion of an initially pressure less, constant-density, gamma-law gas due to a convergent velocity field. A strong shock front converges at the 

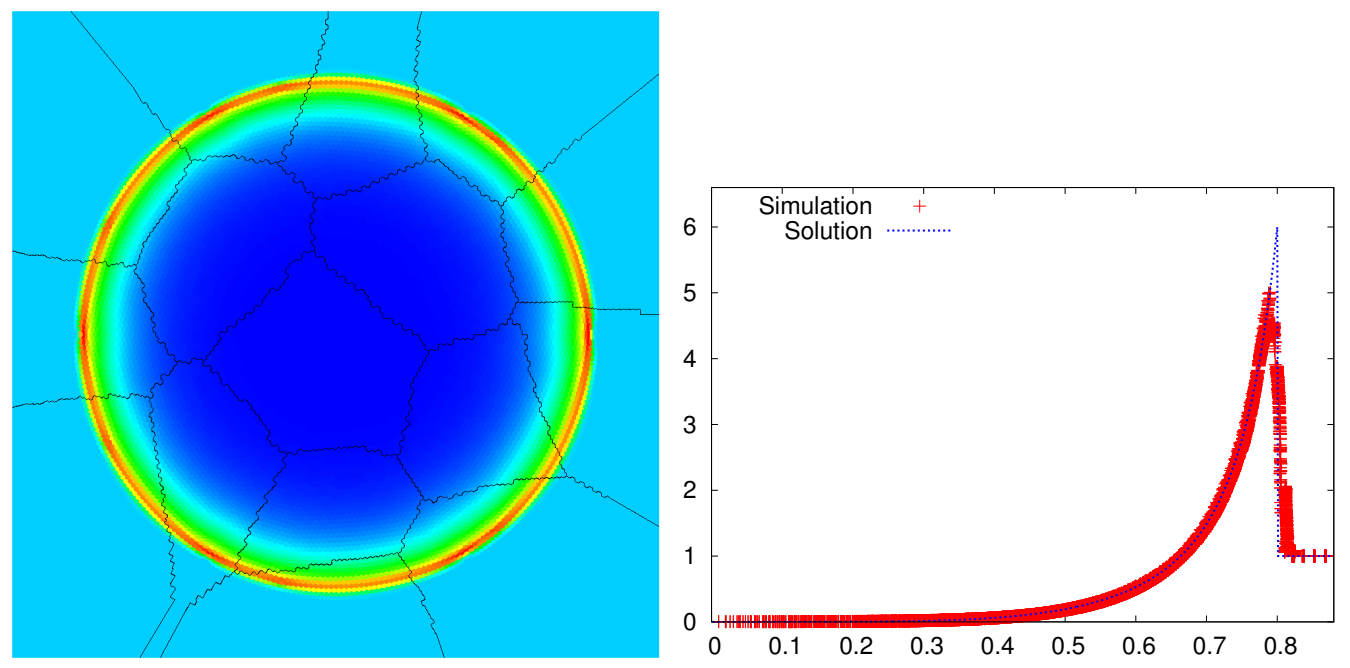

Figure 24: Sedov problem on radial Voronoi mesh with $N_{r}=64$. Zonal density using variation-diminishing reconstruction with harmonic average reference.

center of the domain, rebounds, and reflects back towards the boundary. As with Sedov, exact similarity solutions exist for planar (1D), cylindrical (2D), and spherical (3D) flows.

We initialize the problem with a zero-pressure ideal gas having $\gamma=5 / 3$ and unit initial density. A convergent velocity field is initialized with unit initial magnitude. The initial velocity $\mathbf{u}_{0}$ at point $\mathbf{x}$ is given by

$$
\mathbf{u}_{0}=\frac{\mathbf{x}-\mathbf{x}_{c}}{\left\|\mathbf{x}-\mathbf{x}_{c}\right\|}
$$

where $\mathbf{x}_{c}$ denotes the center of the problem domain. This yields a peak shock density of 16 at the center of the problem. We compute numerical solutions on two separate grids: a regular $N \times N$ lattice and a radially-symmetric unstructured grid of arbitrary polygons. The regular lattice domain is a unit square with center point $(0.5,0.5)$. We run to final time 0.34 to avoid interactions between the reflected shock front and in-falling outer boundary. The polygonal mesh domain is a circle centered at $(0,0)$ with radius 1 . The final time is 0.6. As with the Sedov tests, the reconstruction algorithm preserves symmetry in all test cases. 

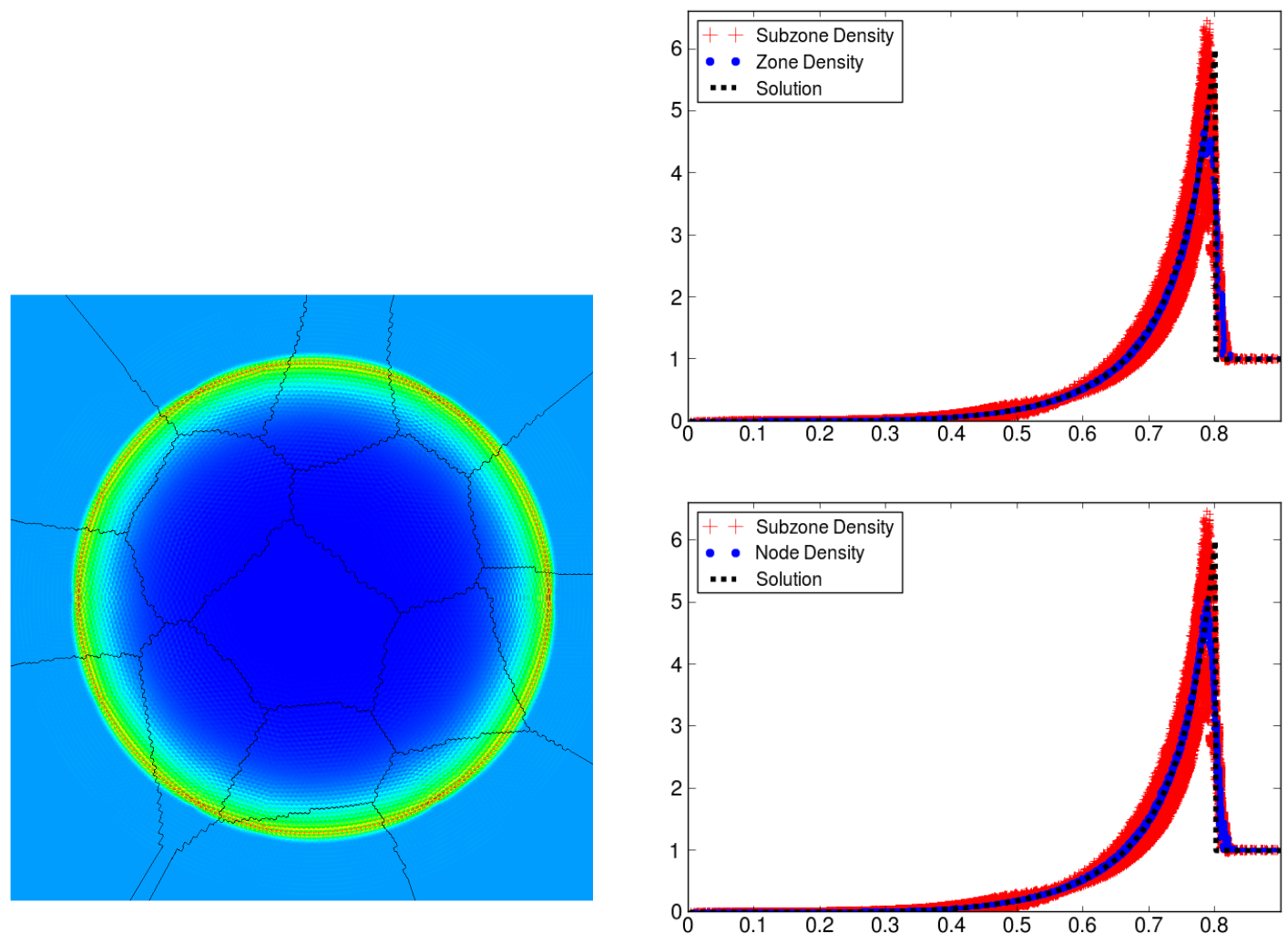

Figure 25: Sedov problem on radial Voronoi mesh with $N_{r}=64$. Reconstructed subzonal density with harmonic average reference. Corresponding nodal and zonal densities are overlaid.

\section{D Noh Problem on a Regular Grid}

The solution to the Noh problem is not as sensitive to momentum conservation errors as was demonstrated in the Sedov blast wave. Figure 27 compares the spatial profiles with flattened (top) and reconstructed (bottom) subzonal densities on a mesh of $128^{2}$ zones. Although linear momentum conservation is not enforced in the top row of solutions, it nevertheless captures the correct shock position and post-shock state. The solution with subzonal reconstruction also captures the correct shock position, but underestimates the post-shock density compared to the flattened solution. Note that the density feature between radii 0.15 and 0.2 corresponds to the boundary of 

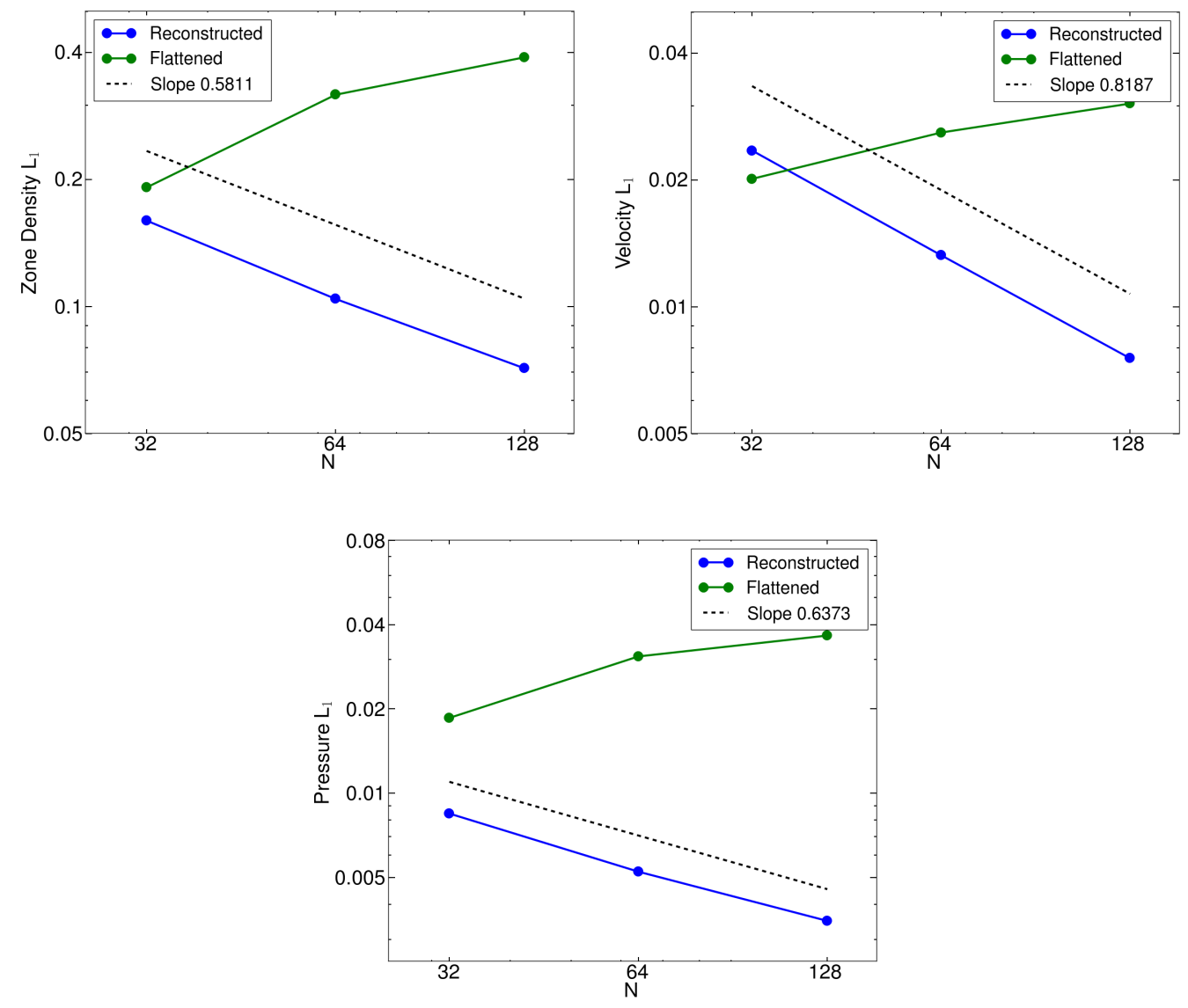

Figure 26: Sedov problem on a radial Voronoi mesh. Plotted are $L_{1}$ convergence in zone density, radial velocity, and pressure comparing a flattened reconstruction to a variationdiminishing reconstruction with harmonic average reference.

the (square) domain along the cardinal axes.

Both the flattened and reconstructed Noh solutions converge at first order under grid refinement. Figure 28 demonstrates this for density, pressure, and radial velocity. Absolute errors are slightly smaller for the flattened solution, further highlighting the fact that momentum conservation does not play as large a role for the Noh test problem. 

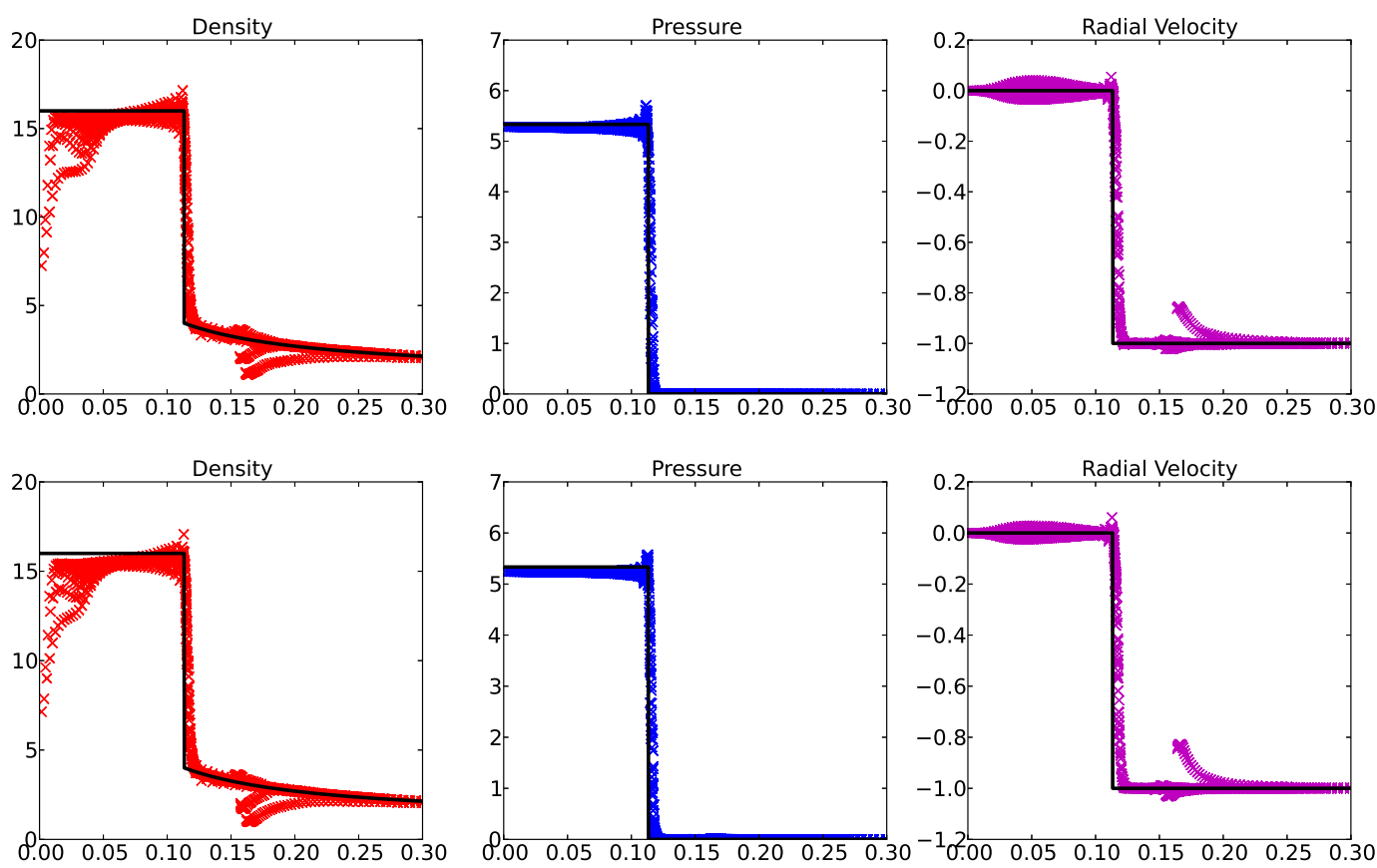

Figure 27: Noh problem on a mesh of $128^{2}$ quads: radial profiles of zone density, pressure, and radial velocity comparing a nonconservative, flattened reconstruction (top row) to a conservative reconstruction with harmonic average reference (bottom row).

\section{D Noh Problem on an Unstructured Polygonal Grid}

Similar to the Cartesian grid case, we observe excellent agreement with the analytic solution for both flattened and reconstructed subzonal densities (see fig. 29). Although the reconstructed solution conserves mass, momentum, and total energy to roundoff, the shock front appears ahead of the analytic position. The flattened solution does not conserve momentum but appears to capture the correct shock position.

Figure 30 establishes grid convergence on a radial polygonal mesh. As with the Cartesian grid case, the nonconservative, flattened solution produces smaller absolute errors. The convergence rate appears larger for the flattened solution when going from the lowest resolution (20 radial zones) to the middle resolution (40 radial zones); however the rate matches that of the conservative reconstructed solution when going to the highest resolution (80 radial zones). 


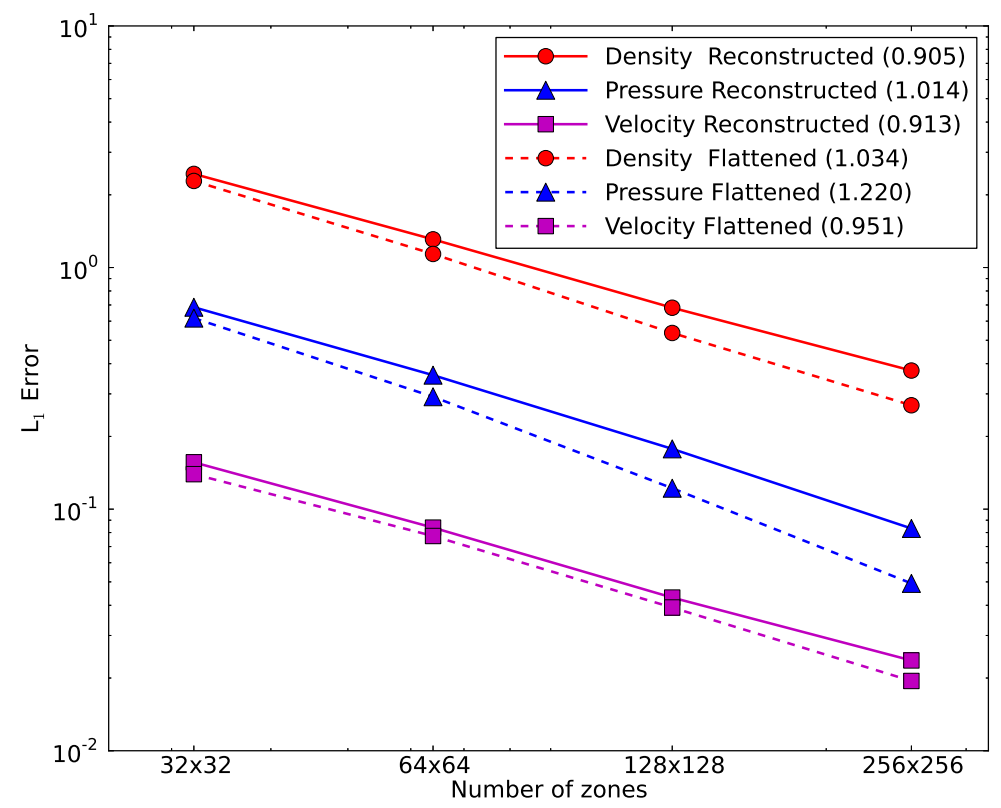

Figure 28: Noh problem on a quad mesh: $L_{1}$ convergence in zone density, pressure, and radial velocity comparing a nonconservative, flattened reconstruction (dashed) to a conservative reconstruction (solid) with harmonic average reference.

\section{Conclusions}

We have introduced a new framework for reconstructing subzonal quantities within a staggered discretization following an overlay-based remap. Examples focus on reconstructing subzonal density to enforce compatibility between zonal and nodal representations of mass. The driving principle of this derivation is maintaining conservation of linear momentum in staggered Lagrangian hydrodynamics.

Numerical results are very favorable. In pure remapping tests, the algorithm capably reconstructs smooth and discontinuous fields on both structured and unstructured meshes. In hydrodynamic tests, we demonstrate improved shock capturing due to exact momentum conservation. Convergence in $L_{1}$ with respect to mesh resolution is established and meets expectations.

It is possible for the reconstruction algorithm as posed to compute neg- 

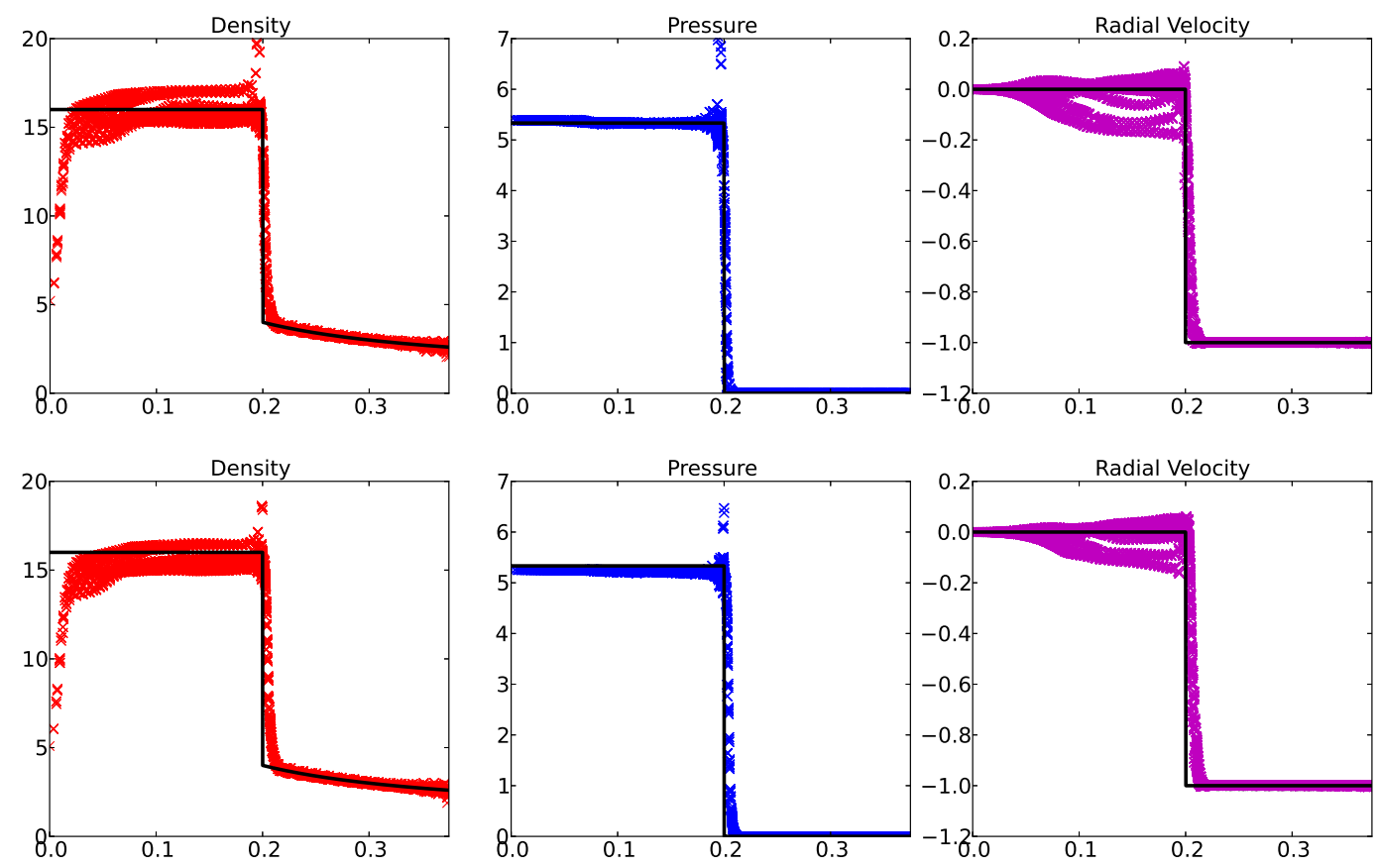

Figure 29: Noh problem on a mesh of 18961 arbitrary polygons: radial profiles of zone density, pressure, and radial velocity comparing a nonconservative, flattened reconstruction (top row) to a conservative reconstruction with harmonic average reference (bottom row).

ative subzonal values, which are invalid as mass densities. Test cases with arbitrarily large density discontinuities were shown to produce such negative densities for certain choices of the reference density. The harmonic average outperformed all other references in pure remapping tests due to its robustness to negative reconstructions and its established second-order convergence for smooth fields.

A local procedure has been presented for repairing negative reconstructed states if and when they occur. Local repair is not strictly conservative at nodes; however, the errors are small, carry no definite sign, and do not impact momentum conservation if velocity is constant. We further note that hydrodynamics tests are less likely to require repair than pure remapping problems as the steep discontinuities found to trigger negative reconstructions are smooth over several grid cells due to numerical diffusion. A globally-coupled 


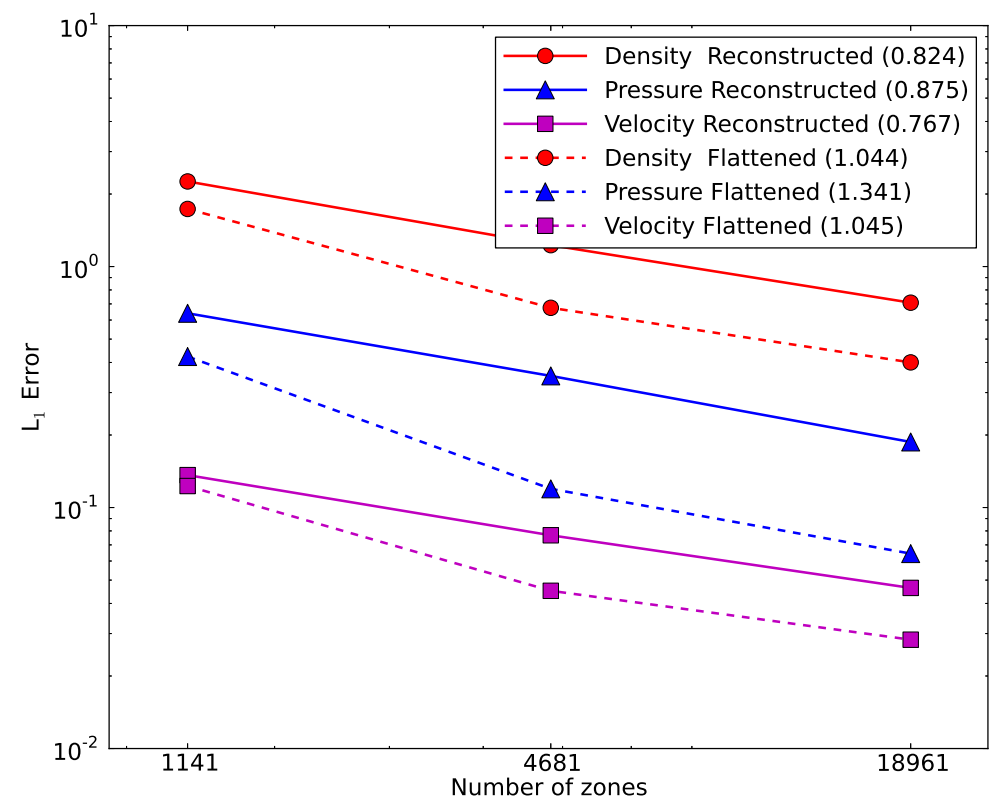

Figure 30: Noh problem on a quad mesh: $L_{1}$ convergence in zone density, pressure, and radial velocity comparing a nonconservative, flattened reconstruction (dashed) to a conservative reconstruction (solid) with harmonic average reference.

and conservative strategy for repairing negative states is also offered as a source of future investigations.

\section{Future Work: Generalization to Subzonal Temperatures}

The problem of staggered-grid overlay remapping is both challenging and fruitful. The results of this article demonstrate the reconstruction algorithm performs well for single-material ALE hydrodynamics. We suggest a number of extensions and improvements here as sources of future work.

Extensions of the algorithm will focus on multi-material remapping, multiphysical remapping, and 3D. One of the motivations for reconstructing subzonal quantities as opposed to directly remapping them as in [14], is that the direct remap lacks a multi-material extension. An algorithm for reconstructing multi-material subzonal masses has been implemented and will constitute 
a follow-on to this article.

Extending the algorithm to subzonal quantities other than mass is necessary for solving multi-physical problems. In the case of spatially-staggered FLD radiation transport, a subzonal description of the material temperature is supplied to improve the accuracy of radiation-fluid coupling terms [25, 26]. The goal of reconstruction in this instance is the preservation of subzonal temperature variation through an overlay remap while maintaining thermal energy conservation. For instance, the thermal energy per subzone is given by $\epsilon_{c}=V_{c} C_{V_{c}} T_{c}$, where $T_{c}$ is the subzonal temperature and $C_{V_{c}}$ is the heat capacity per unit volume. As with mass, zonal and nodal representations of $\epsilon$ are defined by summing local subzones. The overlay then remaps the zonal and nodal thermal energies. The reconstruction stage proceeds as before by solving (23), with mass $m$ replaced by thermal energy $\epsilon$. An optimal reference temperature must be specified to define (6).

Likewise, improvements to the algorithm will focus on repairing negative states, improving monotonicity, and enforcing inequality constraints during the optimization procedure. We suggest two options in the appendices.

Appendix B outlines a globally-coupled, conservative procedure for repairing negative or non-monotonic reconstructions. Pure remapping experiments involving steep density discontinuities led to over-constrained systems of equations, ultimately requiring local, nonconservative repair. Further investigation is needed to determine viability of the global algorithm.

Appendix C generalizes the constrained optimization problem (7) to include inequality constraints. Such constraints may directly enforce positivity or monotonicity of the reconstructed subzonal field without the need for repair. The framework of quadratic programming provides a path forward for investigating these ideas further.

Overall, we view the problem of reconstructing subzonal quantities that preserve monotonicity to be a high priority. Although the staggered compatible hydro algorithm [2] used in this paper is insensitive to non-monotonicities in the subzonal density field, the same cannot be true for subzonal temperatures in staggered-grid rad-hydro. Oscillations in temperature at subzones can trigger spurious emission/absorption sources and possible disrupt states of thermodynamic equilibrium.

\section{Acknowledgments}

This work was performed under the auspices of the U. S. Department of Energy by Lawrence Livermore National Laboratory under Contract DE- 


\section{AC52-07NA27344.}

[1] P. Anninos, Kull ALE: II. Grid Motion on Unstructured Arbitrary Polyhedral Meshes, Technical Report: UCRL-ID-147297-PT-2, Lawrence Livermore National Laboratory, 2002.

[2] E. Caramana, D. Burton, M. Shashkov, P. Whalen, The Construction of Compatible Hydrodynamics Algorithms Utilizing Conservation of Total Energy, Journal of Computational Physics, 146, pp. 227-262, 1998.

[3] B. Despres, R. Loubère Convergence and Sensitivity Analysis of Repair Algorithms in 1D, International Journal on Finite Volumes, 2006.

[4] D. Flanagan and Belytschko T. Uniform Strain Hexahedron and Quadrilateral with Orthogonal Hourglass Control, International Journal on Numerical Methods in Engineering, 17(5), pp. 679-706, 1981.

[5] J. Gondzio, Multiple Centrality Corrections in a Primal-Dual Method for Linear Programming, Computational Optimization and Applications, 6, pp. 137-156, 1996.

[6] J. Grandy, Conservative Remapping and Region Overlays by Intersecting Arbitrary Polyhedra, Journal of Computational Physics, 148, pp. 433-466, 1999.

[7] T. Harribey, J. Breil, P.-H. Maire, M. Shashkov, A Swept-IntersectionBased Remapping Method in a ReALE Framework International Journal for Numerical Methods in Fluids, 72(6), pp. 697-708, 2013.

[8] hypre. High performance preconditioners. http://www.llnl.gov/CASC/linearsolvers/

[9] W. Karush, Minima of Functions of Several Variables with Inequalities as Side Constraints, MS Dissertation, Department of Mathematics, University of Chicago, 1939.

[10] M. Kucharik and M. Shashkov, Extension of Efficient, SweptIntegration-Based Conservative Remapping Method for Meshes with Changing Connectivity, International Journal for Numerical Methods in Fluids, 56, pp. 1359-1365, 2007. 
[11] H. Kuhn and A. Tucker, Nonlinear Programming, Proceedings of 2nd Berkeley Symposium on Mathematical Statistics and Probability, pp. 481-492, 1951.

[12] R. Liska, M. Shashkov, P. Vachal, B. Wendroff, Optimization-Based Synchronized Flux-Corrected Conservative Interpolation (Remapping) of Mass and Momentum for Arbitrary Lagrangian-Eulerian Methods, Journal of Computational Physics, 229, pp. 1467-1497, 2010.

[13] R. Loubère, P.-H. Maire, M. Shashkov, J. Breil, S. Galera, ReALE: A Reconnection-Based Arbitrary Lagrangian-Eulerian Method, Journal of Computational Physics, 229, pp. 4724-4761, 2010.

[14] R. Loubère and M. Shashkov, A Subcell Remapping Method on Staggered Polygonal Grids for Arbitrary Lagrangian-Eulerian Methods, Journal of Computational Physics, 209, pp. 105-138, 2005.

[15] R. Loubère and E. Caramana, The Force/Work Differencing of Exceptional Points in the Discrete Compatible Formulation of Lagrangian Hydrodynamics, Journal of Computational Physics, 216(1), pp. 1-18, 2006.

[16] R. Loubère, M. Staley, B. Wendroff The Repair Paradigm: New Algorithms and Applications to Compressible Flow, Journal of Computational Physics, 211(2), pp. 385-404, 2006.

[17] R. Loubère Contribution to the Lagrangian and ALE Numerical Methods, Habilitation à Diriger des Recherches, University of Toulouse, 2013.

[18] L. Margolin and M. Shashkov, Remapping Recovery and Repair on a Staggered Grid, Comput. Methods Appl. Engrg., 193, pp. 4139-4155, 2004.

[19] S. Mehrotra, On the Implementation of a Primal-Dual Interior Point Method, SIAM Journal on Optimization, 2, pp. 576-601, 1992.

[20] W. Noh, Errors for Calculations of Strong Shocks Using an Artificial Viscosity and an Artificial Heat Flux, Journal of Computational Physics, 72, pp. 78-120, 1987.

[21] J. M. Owen, M. Shashkov, Arbitrary Lagrangian Eulerian Remap Treatments Consistent with Staggered Compatible Total Energy Conserving 
Lagrangian Methods, Journal of Computational Physics, 273, pp. 520$547,2014$.

[22] L. Sedov, Propagation of Strong Shock Waves, Journal of Applied Mathematics and Mechanics, v. 10, pp. 241-250, 1946.

[23] L. Sedov, Similarity and Dimensional Methods in Mechanics, Academic Press, New York, 1959.

[24] J Lockwood-Taylor, An Exact Solution of the Spherical Blast Wave Problem, Philosophical Magazine, v. 46, pp. 317-320, 1955.

[25] M. Adams, Subcell Balance Methods for Radiative Transfer on Arbitrary Grids, Transport Theory and Statistical Physics, 26, pp. 385-431, 1997.

[26] T. Eaton, M. Adams, A New Corner-Balance/Linear-Discontinuous Method for Transport in Slab Geometry, Transactions of the American Nuclear Society, 70, 158, 1994.

[27] T. Kaiser, Evolution of a Zone-Centered Field with a Node-Centered Diffusion Solver on an Unstructured Mesh, American Physical Society, 44th Annual Meeting of the Division of Plasma, abstract \#BP1.109, 2002 .

[28] T. Palmer, Discretization of the Diffusion Equation on Unstructured Polygonal Meshes in Two Dimensions, Annals of Nuclear Energy, 28, pp. 1851, 2001.

[29] T. Bailey, M. Adams, B. Yang, M. Zika, A Piecewise Linear Finite Element Discretization of the Diffusion Equation for Arbitrary Polyhedral Grids, Mathematics and Computation, Supercomputing, Reactor Physics and Nuclear and Biological Applications Conference (ANS), 2005. 


\section{A. Analysis of the Singular Matrix}

Consider the linear system (23). The matrix $\mathbb{A}$ may be written

$$
\mathbb{A}=\mathbb{V}_{c n}^{T}\left(\mathbb{I}-\mathbb{V}_{c z}\left(\mathbb{V}_{c z}^{T} \mathbb{V}_{c z}\right)^{-1} \mathbb{V}_{c z}^{T}\right) \mathbb{V}_{c n}
$$

Without loss of generality, we have replaced the diagonal matrix of positive reference values $\mathbb{M}_{c}$ with the identity matrix. We wish to explore the null space of $\mathbb{V}$ through its sub-matrices. Let

$$
\mathbb{P}=\mathbb{V}_{c z}\left(\mathbb{V}_{c z}^{T} \mathbb{V}_{c z}\right)^{-1} \mathbb{V}_{c z}^{T}
$$

It is easy to check that the matrix $\mathbb{P}$ is a projector (i.e. $\mathbb{P}^{2}=\mathbb{P}$ ). The form of (A.2) implies that $\mathbb{P}$ is an orthogonal projector onto the range of matrix $\mathbb{V}_{c z}$. In addition,

$$
\mathbb{Q}=\mathbb{I}-\mathbb{P}
$$

is a projector orthogonal to $\mathbb{P}$ : the range of $\mathbb{Q}$ is the null space of $\mathbb{P}$ and vice versa.

Because each corner $c$ corresponds to a unique zone $z$ and a unique node $n$, each row of $\mathbb{V}_{c z}$ and $\mathbb{V}_{c n}$ has exactly one nonzero element, implying the two matrices are full-rank. Therefore, the null space of

$$
\mathbb{V}=\mathbb{V}_{c n}^{T} \mathbb{Q} \mathbb{V}_{c n}
$$

is equal in size to the null space of $\mathbb{Q}$. It remains to find the null space of $\mathbb{Q}$.

Let $\mathbf{x} \in \operatorname{null}(\mathbb{A})$, then there exists some $\mathbf{w} \in \operatorname{range}\left(\mathbb{V}_{c n}\right)$ such that $\mathbf{w}=$ $\mathbb{V}_{c n} \mathbf{x}$ and $\mathbb{Q} \mathbf{w}=0$. We note

(i) $\mathbb{Q}$ and $\mathbb{P}$ are orthogonal: $\mathbf{w} \in \operatorname{null}(\mathbb{Q}) \Longrightarrow \mathbf{w} \in \operatorname{range}(\mathbb{P})$.

(ii) $\mathbb{P}$ projects onto the range of $\mathbb{V}_{c z}$.

Using $(i)$ and $(i i)$, there must exist $\mathbf{y} \in \operatorname{range}\left(\mathbb{V}_{c z}\right)$ such that $\mathbf{w}=\mathbb{V}_{c z} \mathbf{y}$. Finally, for $\mathbf{x}$ to be in the null space of $\mathbb{A}$, there must exist $\mathbf{y}$ such that

$$
\mathbb{V}_{c z} \mathbf{y}=\mathbb{V}_{c n} \mathbf{x}
$$

We wish to show that the space spanned by $\mathbf{x}$ satisfying (A.4) is of dimension 1. We propose this space consists of the set of constant vectors. Geometrically, this is plausible: the action of $\mathbb{V}_{c n}$ takes node-centered data 
and projects it to its corners (weighted by corner volume). Similarly for $\mathbb{V}_{c z}$. Each corner has a unique zone and a unique node. If node-centered data $\mathbf{x}$ and zone-centered data $\mathbf{y}$ satisfy (A.4), the data must be constant.

Proof: Suppose not. Then there exists some non-constant node-centered data $\mathbf{x}$ and zone- centered data $\mathbf{y}$ satisfying (A.4). The $i$-th elements of $\left(\mathbb{V}_{c n} \mathbf{x}\right)$ and $\left(\mathbb{V}_{c z} \mathbf{y}\right)$ correspond to corner $c_{i}$ :

$$
\left(\mathbb{V}_{c n} \mathbf{x}\right)_{i}=V_{c_{i}} x_{n_{c_{i}}} \quad \text { and } \quad\left(\mathbb{V}_{c z} \mathbf{x}\right)_{i}=V_{c_{i}} y_{z_{c_{i}}} .
$$

For (A.4) to hold, $x_{n_{c}}=y_{z_{c}}$ at every corner $c$. In other words, the values $y_{z}$ for zones around node $n$ must be equal to $x_{n}$, and the values $x_{n}$ for nodes around zone $z$ must be equal to $y_{z}$. For this to hold, both $x_{n}$ and $y_{z}$ must take on the same value at every node and zone respectively. Therefore, only constant data may satisfy $\mathbf{x} \in \operatorname{null}(\mathbb{A})$ and (A.4).

\section{B. Global Repair of Negative Reconstructed Densities}

We generalize the optimization problem in section 2 to include additional constraints at subzones. More precisely, we augment (7) with equality constraints at a subset of subzones:

$$
\begin{array}{ll}
\text { Given: } & \rho_{z}, \rho_{n}, V_{c} \\
\text { Minimize: } & \frac{1}{2} \sum_{c}\left(\frac{\rho_{c}-\mu_{c}}{\mu_{c}}\right)^{2} \\
\text { Subject to: } & \begin{cases}\sum_{c(z)} V_{c}\left(\rho_{c}-\rho_{z}\right)=0, & \forall z \\
\sum_{c(n)} V_{c}\left(\rho_{c}-\rho_{n}\right)=0, & \forall n \\
\rho_{c}=\epsilon_{c} \quad \forall c \in \mathcal{C}\end{cases}
\end{array}
$$

Where $\epsilon_{c}$ is the constrained value of $\rho_{c}$, and $\mathcal{C}$ may be any subset of subzones. The system remains under-constrained provided $N_{z}+N_{n}+|\mathcal{C}|<N_{c}$.

We interpret the solution to (B.1) in terms of a repair procedure to correct unsatisfactory values in the solution to (7) (i.e. negatives, non-monotonicities). Let $\rho_{c}^{(0)}$ denote the solution to the original reconstruction problem under some 
arbitrary reference state. We solve (B.1) to obtain the correction $\rho_{c}^{(1)}$, where the reference state is given by the previous solution $\rho_{c}^{(0)}$. (The advantage of choosing the previous solution as the reference state ensures that the reference automatically satisfies conservation.)

The set $\mathcal{C}$ consists of a collection of subzones sharing some undesirable property we wish to repair. For positivity constraints, one may consider the subset

$$
\mathcal{C}=\mathcal{C}_{-}^{(0)}=\left\{c: \rho_{c}^{(0)} \leq 0\right\}
$$

For a stricter constraint such as monotonicity, one may consider

$$
\mathcal{C}=\mathcal{C}_{\text {mono }}^{(0)}=\left\{c: \rho_{c}^{(0)} \notin\left[\min \left(\rho_{z_{c}}, \rho_{n_{c}}\right), \max \left(\rho_{z_{c}}, \rho_{n_{c}}\right)\right]\right\}
$$

For the purposes of this paper, we limit our focus to (B.2) and consider monotonicity constraints to be a source of future work.

Finally, we have freedom to choose the set of constrained subzonal values $\left\{\epsilon_{c}\right\}_{\mathcal{C}}$. For positivity constraints, we select a local positive value for each subzone. One option is to use

$$
\epsilon_{c}=\alpha \min \left(\rho_{z_{c}}, \rho_{n_{c}}\right),
$$

where $0<\alpha \leq 1$. Note that as $\alpha$ approaches 1 , the positivity constraint becomes stricter.

The method of Lagrange multipliers solves (B.1). The Lagrangian is

$$
\begin{aligned}
L\left(\rho_{c}, \lambda_{z}, \lambda_{n}, \lambda_{c}\right)=\frac{1}{2} \sum_{c}\left(\frac{\rho_{c}-\mu_{c}}{\mu_{c}}\right)^{2} & +\sum_{z} \lambda_{z} \sum_{c(z)} V_{c}\left(\rho_{c}-\rho_{z}\right) \\
& +\sum_{n} \lambda_{n} \sum_{c(n)} V_{c}\left(\rho_{c}-\rho_{n}\right), \\
& +\sum_{c} \lambda_{c} \chi_{c} V_{c}\left(\rho_{c}-\epsilon_{c}\right)
\end{aligned}
$$

where $\lambda_{c}$ denotes the Lagrange multipliers for the subzonal constraints and $\chi_{c}$ is an indicator function that is 1 for $c \in \mathcal{C}$ and 0 otherwise. Formulas (11) and (15) for the nodal and zonal Lagrange multipliers hold, with $w_{c}, \Delta m_{z}$, 
and $\Delta m_{n}$ replaced by

$$
\begin{aligned}
& w_{c}=\left(\mu_{c} V_{c}\right)^{2}\left(1-\chi_{c}\right) \\
& \Delta m_{z}=m_{z}-\sum_{c(z)}\left(\left(1-\chi_{c}\right) \mu_{c}+\chi_{c} \epsilon_{c}\right) V_{c} . \\
& \Delta m_{n}=m_{n}-\sum_{c(n)}\left(\left(1-\chi_{c}\right) \mu_{c}+\chi_{c} \epsilon_{c}\right) V_{c}
\end{aligned}
$$

The linear system (23) is modified

$$
\mathbb{A}=\mathbb{V}_{c n}^{T} \mathbb{M}_{c}\left(\mathbb{I}_{c}-\mathbb{X}_{c}\right)\left(\mathbb{I}_{c}-\mathbb{M}_{c} \mathbb{V}_{c z} \mathbb{W}_{z}^{-1} \mathbb{V}_{c z}^{T} \mathbb{M}_{c}\right)\left(\mathbb{I}_{c}-\mathbb{X}_{c}\right) \mathbb{M}_{c} \mathbb{V}_{c n},
$$

where $\mathbb{X}_{c}=\operatorname{diag}\left(\chi_{c}\right)$. The updated subzonal densities are

$$
\rho_{c}=\left(1-\chi_{c}\right) \mu_{c}+\chi_{c} \epsilon_{c}-V_{c} \mu_{c}^{2}\left(1-\chi_{c}\right)\left(\lambda_{z_{c}}+\lambda_{n_{c}}\right) .
$$

Remark: For problems with steep discontinuities, it is possible that a single global repair step is insufficient to remove all negative subzonal states. When this occurs, an iterative repair may be performed. Given state $\rho_{c}^{(i)}$, we solve (B.1) for new subzonal densities $\rho_{c}=\rho_{c}^{(i+1)}$ using reference $\mu_{c}=\rho_{c}^{(i)}$ and subzone subset

$$
\mathcal{C}=\mathcal{C}_{-}^{(i)}=\left\{c: \rho_{c}^{(j)} \leq 0, \forall j \leq i\right\} .
$$

We define $\mathcal{C}_{-}^{(i)}$ such that, once a subzone is constrained, it is constrained for all iterations to ensure convergence of the iterative repair.

\section{Optimization with Equality and Inequality Constraints: Quadratic Programming Formulation}

The optimization problem (7) may be expressed as a quadratic programming (QP) problem:

$$
\begin{array}{ll}
\operatorname{minimize}: & f(\mathbf{x})=\frac{1}{2} \mathbf{x}^{T} \mathbb{Q} \mathbf{x}+\mathbf{c}^{T} \mathbf{x} \\
\text { subject to: } & \mathbb{A} \mathbf{x}=\mathbf{b} \quad \text { and } \quad \mathbb{C} \mathbf{x} \geq \mathbf{d}
\end{array}
$$


where $\mathbb{Q}$ is a symmetric, positive-definite matrix, c is a constant vector, and $\mathbb{A}$ and $\mathbb{C}$ are full-rank matrices comprising the equality and inequality constraints respectively on the target variable $\mathbf{x}$.

We follow the Karush-Kuhn-Tucker formalism [9, 11] for optimization with mixed constraints. We define Lagrange multipliers $\lambda$ and $\gamma$ corresponding to the equality and inequality constraints respectively. An extra variable s enforces the inequality constraints through complementary slackness.

We solve for fixed points of the Lagrangian

$$
L(\mathbf{x} ; \lambda, \gamma, \mathbf{s})=\frac{1}{2} \mathbf{x}^{T} \mathbb{Q} \mathbf{x}+\mathbf{c}^{T} \mathbf{x}+\lambda^{T}(\mathbb{A} \mathbf{x}-\mathbf{b})+\gamma^{T}(\mathbb{C} \mathbf{x}-\mathbf{s}-\mathbf{d}) .
$$

satisfying complementarity condition

$$
\gamma_{i} s_{i}=0, \quad \gamma_{i} \geq 0, \quad s_{i} \geq 0
$$

for each inequality constraint $i$. Solving for the fixed points of (C.2) corresponds to solving the following coupled systems:

$$
\begin{aligned}
\mathbf{c}+\mathbb{Q} \mathbf{x}-\mathbb{A}^{T} \lambda-\mathbb{C}^{T} \gamma & =0 \\
\mathbb{A} \mathbf{x}-\mathbf{b} & =0 \\
\mathbb{C} \mathbf{x}-\mathbf{s}-\mathbf{d} & =0 \\
\mathbb{S} \Gamma \mathbb{1}_{e} & =0 \\
\mathbf{s}, \gamma & \geq 0
\end{aligned}
$$

where $\mathbb{S}=\operatorname{diag}(\mathbf{s}), \Gamma=\operatorname{diag}(\gamma)$, and $\mathbb{1}_{e}$ denotes a vector of ones.

For the problem of subzonal reconstruction, we have convenient forms for the coefficients of (C.4). The target variable is the relative variation with respect to reference $\mu$ per subzone:

$$
x_{c}=\frac{\rho_{c}-\mu_{c}}{\mu_{c}}, \quad c=1, \ldots, N_{c}
$$

The objective function simplifies to

$$
\mathbb{Q}=\mathbb{I}_{c} \quad \text { and } \quad \mathbf{c}=0
$$

Zonal (4) and nodal (5) equality constraints are written compactly

$$
\lambda=\left[\begin{array}{c}
\lambda_{z} \\
\lambda_{n}
\end{array}\right], \quad \mathbb{A}=\left[\begin{array}{c}
\mathbb{V}_{c z}^{T} \mathbb{M}_{c} \\
\mathbb{V}_{c n}^{T} \mathbb{M}_{c}
\end{array}\right], \quad \mathbf{b}=\left[\begin{array}{c}
\mathbf{m}_{z}-\mathbb{V}_{c z}^{T} \mu \\
\mathbf{m}_{n}-\mathbb{V}_{c n}^{T} \mu
\end{array}\right],
$$


where $\lambda$ is length $N_{z}+N_{n}$.

The inequality constraints may be used to enforce bounds on the reconstructed variables. Enforcing one-sided bounds for $\left\{\rho_{c}\right\}_{c}$ of the form $\rho_{c} \geq \rho_{c}^{\min }$ corresponds to $N_{c}$ inequality constraints of the form

$$
\mathbb{C}=\mathbb{I}_{c}, \quad \mathbf{d}=\mathbb{M}_{c}^{-1}\left(\rho^{\text {min }}-\mu\right) .
$$

Likewise, two-sided bounds of the form $\rho_{c}^{\text {min }} \leq \rho_{c} \leq \rho_{c}^{\max }$ corresponds to $2 N_{c}$ inequality constraints of the form

$$
\gamma=\left[\begin{array}{c}
\gamma^{\min } \\
\gamma^{\max }
\end{array}\right], \quad \mathbb{C}=\left[\begin{array}{r}
I_{c} \\
-I_{c}
\end{array}\right], \quad \mathbf{d}=\left[\begin{array}{c}
\mathbb{M}_{c}^{-1}\left(\rho^{\min }-\mu\right) \\
-\mathbb{M}_{c}^{-1}\left(\rho^{\max }-\mu\right)
\end{array}\right] .
$$

Enforcing (C.7) is useful for eliminating negative subzonal reconstruction values. Enforcing the stricter constraints in (C.8) may be used to preserve monotonicity locally.

An iterative interior-point method may be used to solve (C.1). Given initial state $\mathbf{v}^{(i)}$ for $\mathbf{v} \in\{\mathbf{x}, \lambda, \gamma, \mathbf{s}\}$ satisfying $\gamma_{i}^{(i)}>0$ and $s_{i}^{(i)}>0$, we solve for step lengths $\Delta \mathbf{v}$ such that $\mathbf{v}+\Delta \mathbf{v}$ solves (C.4a)- (C.4c) and satisfies (C.4e):

$$
\left[\begin{array}{rrrr}
\mathbb{Q} & -\mathbb{A}^{T} & -\mathbb{C}^{T} & 0 \\
\mathbb{A} & 0 & 0 & 0 \\
\mathbb{C} & 0 & 0 & -\mathbb{I} \\
0 & 0 & \mathbb{S} & \Gamma
\end{array}\right]\left[\begin{array}{c}
\Delta \mathbf{x} \\
\Delta \lambda \\
\Delta \gamma \\
\Delta \mathbf{s}
\end{array}\right]=-\left[\begin{array}{c}
\mathbf{r}_{\text {var }} \\
\mathbf{r}_{\text {eq }} \\
\mathbf{r}_{\text {ineq }} \\
\mathbf{r}_{\text {slack }}
\end{array}\right]
$$

Using current data $\mathbf{v}^{(i)}$, the residuals are computed

$$
\begin{aligned}
\mathbf{r}_{\text {var }} & =\mathbb{Q} \mathbf{x}^{(i)}+\mathbf{c}-\mathbb{A}^{T} \lambda^{(i)}-\mathbb{C}^{T} \gamma^{(i)} \\
\mathbf{r}_{\text {eq }} & =\mathbb{A} \mathbf{x}^{(i)}-\mathbf{b} \\
\mathbf{r}_{\text {ineq }} & =\mathbb{C} \mathbf{x}^{(i)}-\mathbf{s}^{(i)}-\mathbf{d} \\
\mathbf{r}_{\text {slack }} & =\mathbb{S}^{(i)} \Gamma^{(i)} \mathbb{1}_{e}
\end{aligned}
$$

corresponding to fixed points of the Lagrangian in the solution variable, equality multipliers, inequality multipliers, and slackness condition respectively. Step lengths are limited by positive factor $\alpha^{(i)}$ such that complementarity holds:

$$
0<\alpha^{(i)} \leq \max _{\alpha}\left\{\gamma^{(i)}+\alpha \Delta \gamma^{(i)}>0, \mathbf{s}^{(i)}+\alpha \Delta \mathbf{s}^{(i)}>0\right\}
$$


The state update is then $\mathbf{v}^{(i+1)}=\mathbf{v}^{(i)}+\alpha^{(i)} \Delta \mathbf{v}^{(i)}$. Iteration continues until the residual vector falls below a given tolerance.

Remark: The system (C.9) must be solved each iteration with new coefficients and residuals. This is the most computationally intensive part of the fixed-point method. Efficient versions, such as [5, 19], incorporate multiple sub-steps by applying higher-order corrections to the minimization path. The coefficient matrix remains fixed during the entire step; it is typically factored and applied to a different residual for each sub-step. Significant savings in numbers of iterations to convergence have been demonstrated.

Remark: System (C.9) may be factored to produce a single linear system of size $N_{z}$ for the zonal equality Lagrange multiplier step lengths. The factored system consists of an $N_{z} \times N_{z}$ sparse matrix identical in structure to (23) described in section 2 . The step lengths of the remaining state variables are obtained through simple linear transformations. 